\title{
QUANTIZATION ON A LIE GROUP: HIGHER-ORDER POLARIZATIONS *
}

\author{
V. Aldaya ${ }^{1,2}$, J. Guerrero ${ }^{1,3}$, and G. Marmo ${ }^{3}$
}

August 27, 2018

\section{Introduction}

Much effort has been devoted to the geometrization of Quantum Mechanics during the second half of this Century in an attempt to emulate Classical Mechanics and Classical Gravity at mathematical beauty and, why not, to better understand Quantum Gravity. We wish to report on one particular line of this task, which lies mostly on symmetry grounds and has been developed in the last years trying to accomodate modern aspects of Quantum Mechanics such as global quantization of systems with non-trivial topology, in particular systems suffering from topological anomalies, and accounting for more general obstructions to the basic rules of local quantization, to be referred to as algebraic anomalies, directly attached to the well-known no-go theorems [1] of the original, Standard Quantum Mechanics. This Group Approach to Quantization (GAQ) [2], in some respect generalizes Geometric Quantization (GQ), originally developed by Kirillov, Kostant and Souriau [3, 4, 5] both as a method of quantization and as a group representation technique, and

\footnotetext{
${ }^{1}$ Instituto Carlos I de Física Teórica y Computacional, Facultad de Ciencias, Universidad de Granada, Campus de Fuentenueva, Granada 18002, Spain

${ }^{2}$ IFIC, Centro Mixto Universidad de Valencia-CSIC, Burjasot 46100-Valencia,Spain.

${ }^{3}$ Dipartimento di Scienze Fisiche and INFN, Sezione di Napoli, Mostra d'Oltremare, Pad. 19, 80125, Napoli, Italia.

*Work partially supported by the Dirección General de Ciencia y Tecnología.
} 
the more specific representation algorithm of Borel-Weyl-Bott (B-W-B) [6], which essentially applies to finite-dimensional semisimple groups.

The idea of turning groups into basic building blocks for the geometric formulation of Physics is simply the natural result of pushing ahead the old usage of imposing the compatibility of observer in the same way Differential Geometry itself considers admissibility of local chart. The requirement of a definite structure in the set of observers, or atlas, delimites seriously the nature of physical laws in that they must be formulated in terms of say $G L(n, R)$-tensors, although this requirement is not restrictive enough so as to actually "predict" dynamical laws. However, the condition of having defined an associative composition law in a set of "active" transformations of a physical system really predicts in many cases its dynamics, and can accordingly be considered as a basic postulate.

In the particular case of Quantum Mechanics the group structure plays a preponderant technical role also because, after all, the quantization map has always been viewed as a representation, in the Lie algebra sense, of definite subalgebras of the general Poisson algebra defined on classical phase space. In this respect, the group manifold along with canonical structures on it will constitute a powerful tool in the practical construction of the quantum representation, mainly due to the existence of two natural, mutually (commuting left and right) group actions. In fact, one of which can be used to compatibly reduce the regular representation (or some generalization of it) given by the other.

However, groups entering the basic postulates of (Quantum) Physics mostly wear a specific topological and algebraic structure which goes around the notion of "extension by $U(1)$ " of both the "classical" symmetry group and the classical phase space, and we wish to dwell a bit on the necessity of this extensions.

To help to visualize how the paper is organized, we give a list of contents:

- Introduction

- Why $S^{1}$-extended phase space?

- Why central extensions of classical symmetries?

- Central extension $\tilde{G}$ of a group $G$

- Group cohomology 
- Cohomology and contractions: Pseudo-cohomology

- Principal bundle with connection $(\tilde{G}, \Theta)$

- Group Approach to Quantization

- U(1)-quantization

- Non-horizontal polarizations

- Simple examples

- The abelian group $R^{k}$

- The semisimple group $S U(2)$

- Algebraic anomalies

- Higher-order polarizations

- The Schrödinger group and Quantum Optics

- The Virasoro group and String Theory

\subsection{Why $S^{1}$-extended phase space?}

In a naive attempt to quantize geometricaly a classical phase space $M$, i.e. to represent irreducibly the Poisson algebra in terms of unitary operators acting on some Hilbert space naturally attached to the manifold $M$, we are primarily aimed to consider the Hamiltonian vector fields $X_{f}$ associated with the function $f$ as a "quantum" operator $\hat{f}$ acting on $C^{\infty}(M)$ by derivation.

In practice, and in the simplest, though rather general, case we start from the space $R \times R^{3} \times R^{3}$ and construct the Poincaré-Cartan form associated with a Hamiltonian function $H$ [7] and its differential, a presymplectic twoform:

$$
\begin{aligned}
\Theta_{P C} & \equiv p_{i} d x^{i}-H d t \\
\Omega & \equiv d \Theta_{P C}=d p_{i} \wedge d x^{i}-\frac{\partial H}{\partial x^{i}} d x^{i} \wedge d t-\frac{\partial H}{\partial p_{i}} d p_{i} \wedge d t
\end{aligned}
$$

$\Omega$ has a one-dimensional kernel generated by $X_{H} \in \operatorname{Ker} d \Theta_{P C}$ such that $d t\left(X_{H}\right)=1$, 


$$
X_{H}=\frac{\partial}{\partial t}+\frac{\partial H}{\partial p_{i}} \frac{\partial}{\partial x^{i}}-\frac{\partial H}{\partial x^{i}} \frac{\partial}{\partial p_{i}},
$$

and the associated equations of motion are the Hamilton equations:

$$
\begin{aligned}
\frac{d t}{d \tau} & =1 \\
\frac{d x^{i}}{d \tau} & =\frac{\partial H}{\partial p_{i}} \\
\frac{d p_{i}}{d \tau} & =-\frac{\partial H}{\partial x^{i}} .
\end{aligned}
$$

The vector field $X_{H}$, if it is complete, defines a one-parameter group $\phi_{\tau}$ which divides the space of movements $R \times R^{3} \times R^{3}$ into classes, $M \equiv$ $\left\{R \times R^{3} \times R^{3}\right\} / \phi_{\tau}$ constitutes the symplectic phase space of the system characterized by the Hamiltonian $H$; the symplectic form obtains by the projection of $\Omega$. The change of variables under which the equations of motion on the quotient become trivial is the Hamilton-Jacobi transformation. As a trivial example let us consider the case $H=\frac{p^{2}}{2 m}$ corresponding to a free particle of mass $m$. In this case the equations for $\phi_{\tau}$ and their inverses are:

$$
\left\{\begin{array} { l } 
{ x ^ { i } = \frac { P ^ { i } } { m } \tau + K ^ { i } } \\
{ p _ { i } = P _ { i } } \\
{ t = \tau }
\end{array} \Longleftrightarrow \left\{\begin{array}{l}
K^{i}=x^{i}-\frac{p^{i}}{m} t \\
P_{i}=p_{i} \\
\tau=t
\end{array}\right.\right.
$$

where the constants of motion $K^{i}, P_{j}$ parametrize the solution manifold $M$. $\Theta_{P C}$ goes to the quotient except for a total differential:

$$
\begin{aligned}
\Theta_{P C} & \rightarrow P_{i} d K^{i}+d\left(\frac{P^{2}}{2 m} \tau\right) \\
\omega & =d P_{i} \wedge d K^{i} .
\end{aligned}
$$

The symplectic form 8, 9] is an anti-symmetric "metric" and defines an isomorphism $\omega^{b}: \mathcal{X}(M) \leftrightarrow \Lambda^{1}(M)$ between the module of vector fields on $M$ and that of one-forms on $M$,

$$
X \in \mathcal{X}(M) \longmapsto \omega(X, \cdot) \equiv i_{X} \omega \in \Lambda^{1}(M)
$$


associating a bracket $\{$,$\} on \Lambda^{1}(M)$ with the Lie bracket of vector fields. In particular, given functions $f, g \in C^{\infty}(M)$, their differentials are associated with Hamiltonian vector fields $X_{f}, X_{g}$. This permits the definition of a Poisson bracket between functions, rather than one-forms, but this time the correspondence $\{,\} \rightarrow[$,$] :$

$$
\left.\{,\}: f, g \longmapsto\{f, g\} / d\{f, g\}=-i_{\left[X_{f}, X_{g}\right.}\right]^{\omega},
$$

is no longer an isomorphism because constant functions have trivial Hamiltonian vector fields. In particular, as regard the example $H=\frac{p^{2}}{2 m}$, and considering $K^{i}, P_{j}$ as the basic coordinates for $M$, we find:

$$
\left\{K^{i}, P_{j}\right\}=\delta_{j}^{i} \cdot 1 \longmapsto\left[X_{K^{i}}, X_{P_{j}}\right]=0
$$

i.e. a Lie algebra homomorphism whose kernel is the central subalgebra of constant functions, $R$, generated by 1 .

We should remark that the correspondence between functions and vector fields can be kept at the movement-space level provided that we restrict ourselves to only those functions which are invariant under the action of $X_{H}$, i.e. pull-backs of functions on $M$.

The existence of a non-trivial kernel in the correspondence between functions and Hamiltonian vector fields is an essential breakdown to the naive geometric approach to quantization ${ }^{\wedge}: f \mapsto \hat{f} \equiv X_{f}$ which would associate the trivial operator to any constant. The simplest way of avoiding this problem will consist in enlarging phase space (and/or movement space) with one extra variable providing one extra component to $X_{f}$, and generalizing accordingly the equation $i_{X_{f}} d \Theta_{P C}=-d f$ so as to get a non-trivial new component even though $f$ is a constant. On a "quantum manifold" $P$ locally isomorphic to $M \times S^{1}$ with "connection form" $\Theta$ such that the curvature two-form $d \Theta$ induces on $M$ a 2 -form equal to the one induced by $\Theta_{P C}$, the equation above can be replaced by the set of equations [0]:

$$
\begin{aligned}
i_{\tilde{X}_{f}} d \Theta & =-d f \\
i_{\tilde{X}_{f}} \Theta & =f
\end{aligned}
$$

generalizing this way the quantization map which now reads (except perhaps for a minus sign) 


$$
\hat{f} \equiv i \tilde{X}_{f}
$$

Locally, we can write $\Theta=\Theta_{P C}+\frac{d z}{i z}, z \in S^{1}$ and then $\tilde{X}_{f}=X_{f}+(f-$ $\left.\Theta\left(X_{f}\right)\right) i z \frac{\partial}{\partial z}$, and we immediately see that (9) have unique solution associating the fundamental (vertical) vector field $\Xi \equiv i z \frac{\partial}{\partial z}$ with the unity of $R$.

The quantization map ${ }^{\wedge}$ is now an isomorphism between the Poisson algebra on $M$ and the Lie subalgebra of vector fields on $P$ that are solutions to (9). For the basic functions we have:

$$
\left\{K^{i}, P_{j}\right\}=\delta_{j}^{i} \cdot 1 \longleftrightarrow\left[\tilde{X}_{K^{i}}, \tilde{X}_{P_{j}}\right]=\delta_{j}^{i} \cdot \Xi
$$

The space of wave functions $\Psi$ is constituted by the complex-valued functions on $P$ which satisfy the $U(1)$-equivariance condition, turning $\Psi$ into a section of an associate bundle of the principal bundle $P \rightarrow M$ [10]:

$$
\Xi \Psi=i \Psi \longleftrightarrow \Psi(K, P, z)=z \Psi(K, P),
$$

on which $\tilde{X}_{f}$ act defining the "quantum" operators.

Unfortunately, the quantization map ^ is faithful but not irreducible as a representation of the Lie algebra of classical functions. At this prequantization level, we are only able to reproduce the Bohr-Sommerfeld-Wilson quantization rules [11]. We know that this representation is reducible because of the existence of non-trivial operators commuting with the basic quantum generators $\hat{K}^{i}, \hat{P}_{i}$. In fact, thinking of the simplest case, that of the free particle for example, and adopting for $\Theta$ the local expression,

$$
\Theta=P_{i} d K^{i}+\frac{d z}{i z},
$$

we get the following basic operators:

$$
\begin{aligned}
\hat{K}^{i} & =i \frac{\partial}{\partial P_{i}}+K^{i} \\
\hat{P}_{j} & =-i \frac{\partial}{\partial K^{j}},
\end{aligned}
$$

and it is clear that the operator $\frac{\partial}{\partial P_{i}}$ does commute with them.

True quantization requires that we determine a subspace of complexvalued functions on which our quantum generators act irreducibly. We must 
then impose a maximal set of mutually compatible conditions in the form $\tilde{X} \Psi=0$, for $\tilde{X}$ in some maximal subalgebra called polarization. For instance, in the example above the operator $\frac{\partial}{\partial P_{i}}$ would become trivial had we selected our subspace by imposing the polarization condition $\frac{\partial}{\partial P_{i}} \Psi=0$, i. e. $\Psi \neq$ $\Psi(P)$. Finding a polarization, however, is not an easy task in general, the reason being that two polarization conditions $\hat{a} \Psi=0, \hat{b} \Psi=0$ would be inconsistent, for instance if $[\hat{a}, \hat{b}]=\hat{1}$. In addition, once a certain polarization has been imposed, the set of physical operators that preserve the polarization is severely restricted.

\subsection{Why central extensions of classical symmetries?}

The close connection between central extensions and quantization is best illustrated by the simple case of the free particle and its semi-invariance properties [12, 13, which has its origin in the specific algebraic structure of the group. Let us dwell on the semi-invariance of the free particle in both classical and quantum versions in $1+1$ dimensions. The most relevant feature is the lack of exact invariance of the Lagrangian

$$
\mathcal{L}=\frac{1}{2} m \dot{x}^{2}
$$

under the Galilean transformations:

$$
\begin{aligned}
x^{\prime} & =x+A+V t \\
t^{\prime} & =t+B .
\end{aligned}
$$

In fact, the transformed Lagrangian acquires an extra term:

$$
\begin{aligned}
\mathcal{L}^{\prime} & =\frac{1}{2} m(\dot{x}+V)^{2}=\frac{1}{2} m \dot{x}^{2}+\frac{1}{2} m V^{2}+m \dot{x} V \\
& =\mathcal{L}+\frac{d}{d t}\left(\frac{1}{2} m V^{2} t+m V x\right)
\end{aligned}
$$

which is a non-zero total derivative not affecting the extremals of the corresponding action. The same phenomenon appears at the infinitesimal level. The infinitesimal generators associated with the finite transformations (16) 
are obtained by deriving the transformed coordinates with respect to each one of the group parameters at the identity $e$ of the group:

$$
\begin{aligned}
X_{B} & =\frac{\partial}{\partial t} \\
X_{A} & =\frac{\partial}{\partial x} \\
X_{V} & =t \frac{\partial}{\partial x} .
\end{aligned}
$$

When we compute the Lie derivative of $\mathcal{L}$ with respect to these generators, i.e. the directional derivative of $\mathcal{L}$ along trajectories of each generator of the group, we find

$$
\begin{aligned}
X_{B} \cdot \mathcal{L} & =0 \\
X_{A} \cdot \mathcal{L} & =0 \\
X_{V} \cdot \mathcal{L} & =\frac{d}{d t}(m x) \neq 0,
\end{aligned}
$$

i.e. the semi-invariance of $\mathcal{L}$ under the boosts comes out again. In a complete equivalent way, the Poincaré-Cartan form (see, e.g. [14]),

$$
\Theta_{P C} \equiv p d x-H d t=\frac{\partial \mathcal{L}}{\partial \dot{x}} d x-(p \dot{x}-\mathcal{L}) d t=\frac{\partial \mathcal{L}}{\partial \dot{x}}(d x-\dot{x} d t)+\mathcal{L} d t
$$

also gives a total differential when the Lie derivative along boots is computed:

$$
L_{X_{V}} \Theta_{P C}=d(m x) .
$$

The quantum Galilean particle suffers from the same pathology although it shows up in a rather different manner. Let us consider the Schrödinger equation:

$$
i \hbar \frac{\partial}{\partial t} \Psi=-\frac{\hbar^{2}}{2 m} \nabla^{2} \Psi
$$

and apply the Galilei transformations (16) to it. The equation (22) acquires an extra term, 


$$
i \hbar \frac{\partial}{\partial t^{\prime}} \Psi+i \hbar V \frac{\partial \Psi}{\partial x^{\prime}}=-\frac{\hbar^{2}}{2 m} \nabla^{\prime 2} \Psi
$$

which can be compensated by transforming the wave function. Allowing for a non-trivial phase factor in front of the transformed wave function of the form

$$
\Psi^{\prime}=e^{\frac{i m}{\hbar}\left(V x+\frac{1}{2} V^{2} t\right)} \Psi
$$

the Schrödinger equation becomes covariant, i.e.

$$
i \hbar \frac{\partial}{\partial t^{\prime}} \Psi^{\prime}=-\frac{\hbar^{2}}{2 m} \nabla^{\prime 2} \Psi^{\prime} .
$$

The need for a transformation like (24) accompanying the space-time transformation (16) to accomplish full invariance strongly suggests the adoption of a central extension of the Galilei group as the basic symmetry for the free particle [15]. The $\hbar$ constant is required to keep the exponent in (24) dimensionless (of course the particular value of $\hbar$ is not fixed by this dimensional argument). At this point it should be remarked that the strict invariance of the classical Lagrangian could also be achieved by a central extension of the Galilei group by the additive group of the real line (a local version of the $\mathrm{U}(1)$ central extension of quantum symmetry). In that case the transformation analogous to (24) should be

$$
S^{\prime}=S+m\left(V x+\frac{1}{2} V^{2} t\right)
$$

where $\mathrm{S}$ is the principal Hamilton function, which wears the dimensions of an action, thus avoiding the need for the fundamental constant $\hbar$ [2].

The successive composition of two transformations in the extended Galilei group $\tilde{G}_{(m)}$ immediately leads to the group law:

$$
\begin{aligned}
B^{\prime \prime} & =B^{\prime}+B \\
A^{\prime \prime} & =A^{\prime}+A+V^{\prime} B \\
V^{\prime \prime} & =V^{\prime}+V \\
\zeta^{\prime \prime} & =\zeta^{\prime} \zeta e^{\frac{i m}{\hbar}\left[A^{\prime} V+B\left(V^{\prime} V+\frac{1}{2} V^{\prime 2}\right)\right]} .
\end{aligned}
$$


The virtue of the central extension is that of making non-trivial the commutator between the generators associated with translations and boosts: $\left[\tilde{X}_{A}, \tilde{X}_{V}\right]=m \Xi$, just mimicking the Poisson bracket between $p$ and $x(K$ and $P$ to be precise) provided that we impose on the wave function the $U(1)$-equivariance condition $\Xi \Psi=i \Psi$. This commutator, responsible of the (Lie-algebra) extension, will dictate in this and more general quantization groups the dynamical character (coordinate-momentum character) of the group parameters [2].

\section{Central extension $\tilde{G}$ of a group $G$}

The case of the $1+1$ extended Galilei group $\tilde{G}_{(m)}$ is an example of a central extension of a group $G$ by $U(1)$. In the general case the group manifold of the central extension $\tilde{G}$ is the direct product $G \times U(1)$, a fact which permits the parametrization of the group by the pair $\tilde{g}=(g, \zeta), g \in G, \zeta \in U(1)$. However, the composition law does not correspond to a direct product of groups. Instead, it is written in the form:

$$
\begin{aligned}
g^{\prime \prime} & =g^{\prime} * g \\
\zeta^{\prime \prime} & =\zeta^{\prime} \zeta e^{i \xi\left(g^{\prime}, g\right)},
\end{aligned}
$$

where $\xi$ is a function with arguments in $G$. In this way, the identification of elements $g \in G$ with $(g, 1) \in \tilde{G}$ will not provide us with a subgroup of $\tilde{G}$. The elements $(e, \zeta)$, where $e$ is the identity in $G$, commute with the whole group, i.e. are central. For (28) to be a group law the function $\xi$ must satisfy

$$
\begin{gathered}
\xi\left(g_{1}, g_{2}\right)+\xi\left(g_{1} * g_{2}, g_{3}\right)=\xi\left(g_{1}, g_{2} * g_{3}\right)+\xi\left(g_{2}, g_{3}\right) \\
\xi(e, g)=0 \quad, \quad \xi(g, e)=0 .
\end{gathered}
$$

However, if there exists a function $\lambda: G \rightarrow R$ such that

$$
\begin{aligned}
\xi\left(g^{\prime}, g\right) & =\lambda\left(g^{\prime} * g\right)-\lambda\left(g^{\prime}\right)-\lambda(g) \\
\lambda(e) & =0
\end{aligned}
$$


we can always resort to a change of variables

$$
\begin{aligned}
& \bar{g}=g \\
& \bar{\zeta}=\zeta e^{-i \lambda(g)},
\end{aligned}
$$

to take (28) to the direct product law $\bar{g}^{\prime \prime}=\bar{g}^{\prime} * \bar{g}, \bar{\zeta}^{\prime \prime}=\bar{\zeta}^{\prime} \bar{\zeta}$.

\subsection{Group cohomology}

The construction above suggests a cohomological structure. A function $\xi: G \times G \rightarrow R$ satisfying (29) is called a two-cocycle, and we say that two two-cocycles are cohomologous if they differ by a coboundary, i.e. a twococycle of the form (30). The function $\lambda$ is said to be the generating function of the coboundary. Central extensions are then characterized by the quotient $H^{2}(G, R)$ of the set, abelian group indeed, of two-cocycles, modulo the subgroup of coboundaries, and is called the $2^{\text {nd }}$ cohomology group of $G$. An equivalence class in $H^{2}(G, R)$ will be denoted by $[[\xi]]$. The general theory of central extensions was formulated in [15] (see also [16, 17]), and a more detailled study of Lie group and Lie algebra cohomology can be found in [2], which includes the connection to symplectic cohomology.

\subsection{Cohomology and contractions: Pseudo-cohomology}

By pseudo-cohomology we generally mean a cohomology subclass $[\xi] \in[[\xi]]$ that can be distinguished inside the trivial cohomology, i.e. selected from the coboundaries, according to some additional structure associated with the group $G$. Pseudo-cohomology phenomenon was firstly studied by [18 and then applied to relativistic quantization in Ref. [19]. The physical origin of pseudo-cohomology can be easily stated as follows. We start from a given group $G$ for which we know a central extension $\tilde{G}$ associated with a twococycle $\xi_{c o b}$ generated by a function $\lambda$ on $G$. Suppose there is a well-defined contraction limit of the group $\tilde{G}$ giving $\tilde{G}_{c}$, in the sense of Inönü and Wigner [20, 21]. This means, in particular, that the two-cocycle $\xi_{c o b}$ is well-behaved under the limit process. But it could well happen that the generating function $\lambda$, however, be ill-defined (infinite) in this limit. Then, the contracted twococycle is no longer a coboundary since there is no $\lambda_{c}$ generating it. The Lie 
algebra structure constant associated with a pseudo-extension, i.e. a central extension characterized by a pseudo-cocyle, really differs from those of the trivial product, a fact which requires the non-triviality of the gradient of $\lambda$ at the identity of the group $G$. In other words, pseudo-cocycles are generated by functions which are, locally, linear functions. For finite-dimensional semisimple groups, for which the Whitehead Lemma applies [22], pseudo-cohomology is absolutely relevant and is also related to the $\breve{C}$ ech cohomology of the generalized Hopf fibration by the Cartan subgroup $H, G \rightarrow G / H$, [23]. In the general case, including infinite-dimensional semisimple Lie groups, for which the Whitehead lemma does not apply, the group law for $\tilde{G}$ will contain twococycles as well as pseudo-cocycles (see [24, 23, 25]). The simplest physical example of a quantum symmetry including such an extension is that of the free non-relativistic particle with spin; the Galilei group must be extended by a true two-cocycle to describe the canonical commutation relations between q's and p's as well as by a pseudo-cocycle associated with the Cartan subgroup of $S U(2)$, to account for the spin degree of freedom [26].

\subsection{Principal bundle with connection $(\tilde{G}, \Theta)$}

Central extensions constitute the simplest, but sufficiently general, examples of joint structures which are basic in the quantization process. In fact, a central extension by $U(1)$ is in turn a Lie group and a $U(1)$ principal bundle on which a connection 1-form can be naturally defined. They are trivial as mere fibre bundle, but not as principal bundles with connection. Of course, more general groups bearing a non-trivial principal bundle structure, with structure group $H$ can be considered, but an appropriate pseudo-extension by a two-cocycle generated by a locally linear function of the fibre parameters provides exactly the same result [23].

The connection 1 -form $\tilde{\theta} \equiv \Theta$ on $\tilde{G}$ will be selected among the components of the left-invariant, Lie algebra-valued, Maurer-Cartan 1-form, in such a way that $\Theta(\Xi)=1$ and $L_{\Xi} \Theta=0$, where $\Xi$ is the vertical (or fundamental) vector field. $\Theta$ will play the role of a connection 1-form associated with the $U(1)$ bundle structure.

If $\tilde{G}$ is the topological product of $G$ and $U(1)$ (as will be always the case in all the examples we are considering), the group law for $\tilde{G}$ can be written as:

$$
\left(g^{\prime}, \zeta^{\prime}\right) *(g, \zeta)=\left(g^{\prime} * g, \zeta^{\prime} \zeta e^{i \xi\left(g^{\prime}, g\right)}\right)
$$


Considering a set of local canonical coordinates at the identity $\left\{g^{i}, i=\right.$ $1, \ldots, \operatorname{dim} G\}$ in $G$, the group law can be given by the set of functions $g^{\prime \prime i}=$ $g^{\prime \prime i}\left(g^{\prime j}, g^{k}, j, k=1, \ldots, \operatorname{dim} G\right)$. We introduce the sets of left- and rightinvariant vector fields of $\tilde{G}$ associated with the set of canonical coordinates $\left\{g^{i}\right\}$ as those which are written as $\frac{\partial}{\partial g^{i}}, i=1, \ldots, \operatorname{dim} G$ at the identity, that is:

$$
\tilde{X}_{g^{i}}^{L}(\tilde{g})=\tilde{L}_{\tilde{g}}^{T} \frac{\partial}{\partial g^{i}}, \quad \tilde{X}_{g^{i}}^{R}(\tilde{g})=\tilde{R}_{\tilde{g}}^{T} \frac{\partial}{\partial g^{i}},
$$

where the tilde refers to operations and elements in $\tilde{G}$. The left- (and right-, since the $\mathrm{U}(1)$ subgroup is central in $\tilde{G}$ ) invariant vector field which at the identity is written as $\frac{\partial}{\partial \zeta}$ is $\Xi(\tilde{g}) \equiv i \zeta \frac{\partial}{\partial \zeta}$. It is the vertical (or fundamental) vector field associated with the fibre bundle

$$
U(1) \rightarrow \tilde{G} \rightarrow G
$$

Analogous considerations can be made for the sets of left- and rightinvariant 1-forms associated with the set of local canonical coordinates $\left\{g^{i}\right\}$, i.e. those which at the identity are written as $d g^{i}$ :

$$
\tilde{\theta}^{L g^{i}}(\tilde{g})=\tilde{L}_{\tilde{g}^{-1}}^{*} d g^{i}=\theta^{L g^{i}}(g), \quad \tilde{\theta}^{R g^{i}}(\tilde{g})=\tilde{R}_{\tilde{g}^{-1}}^{*} d g^{i}=\theta^{R g^{i}}(g) .
$$

For simplicity of notation, we shall omit the point in which the vector fields and 1-forms are calculated. Due to the left and right invariance, we have $\theta^{L g^{i}}\left(\tilde{X}_{g^{j}}^{L}\right)=\delta_{j}^{i}=\theta^{R g^{i}}\left(\tilde{X}_{g^{j}}^{R}\right)$.

We can also compute the left- and right-invariant 1-forms which are dual to the vertical generator $\Xi$ :

$$
\tilde{\theta}^{L \zeta}=\tilde{L}_{\tilde{g}^{-1}}^{*} d \zeta, \quad \tilde{\theta}^{R \zeta}=\tilde{R}_{\tilde{g}^{-1}}^{*} d \zeta .
$$

We shall call $\Theta \equiv \tilde{\theta}^{L \zeta}=\frac{d \zeta}{i \zeta}+\left.\frac{\partial \xi\left(g^{\prime}, g\right)}{\partial g^{i}}\right|_{g^{\prime}=g^{-1}} d g^{i}$ the quantization 1-form. It defines a connection on the fibre bundle $\tilde{G}$ and it is uniquelly determined by the two-cocycle $\xi\left(g_{1}, g_{2}\right)$ (it does not change under changes of local canonical coordinates of $G$ ). Adding to $\xi$ a coboundary $\xi_{\lambda}$, generated by the function $\lambda$, results in a new quantization 1-form $\Theta^{\prime}=\Theta+\Theta_{\lambda}$ with

$$
\Theta_{\lambda}=\lambda_{i}^{0} \theta^{L g^{i}}-d \lambda
$$

where $\left.\lambda_{i}^{0} \equiv \frac{\partial \lambda(g)}{\partial g^{i}}\right|_{g=e}$, that is, the gradient of $\lambda$ at the identity with respect to the local canonical coordinates. Note that $\lambda_{i}^{0}$ are constants, and therefore, 
up to the total diferential $d \lambda, \Theta_{\lambda}$ is left invariant under $G$. We also have $d \Theta_{\lambda}=\lambda_{i}^{0} d \theta^{L g^{i}}$, so that using the relation

$$
d \theta^{L g^{i}}=-\frac{1}{2} C_{j k}^{i} \theta^{L g^{j}} \wedge \theta^{L g^{k}}
$$

where $C_{j k}^{i}, i, j, k=1, \ldots, \operatorname{dim} G$ are the structure constants of the Lie algebra $\mathcal{G} \equiv T_{e} G$ in the basis of the left-invariant vector fields associated with the set of local canonical coordinates $\left\{g^{i}\right\}$, we obtain:

$$
d \Theta_{\lambda}=-\frac{1}{2} \lambda_{i}^{0} C_{j k}^{i} \theta^{L g^{j}} \wedge \theta^{L g^{k}} .
$$

Note that $\lambda$ defines an element $\lambda^{0}$ of the coalgebra $\mathcal{G}^{*}$ of $G$ characterizing the presymplectic form $d \Theta_{\lambda} \equiv d \Theta_{\lambda^{0}}$. It is easy to see then that given $\lambda^{\prime 0}$ and $\lambda^{0}$ on the same orbit of the coadjoint action of $G, \lambda^{\prime 0}=A d(g)^{*} \lambda^{0}$, for some $g \in G$, the corresponding presymplectic forms are related through:

$$
d \Theta_{\lambda^{\prime 0}} \equiv d \Theta_{A d(g)^{*} \lambda^{0}}=A d(g)^{*} d \Theta_{\lambda^{0}} .
$$

Summarizing this section, we can classify the central extensions of $G$ in equivalence classes, using two kinds of equivalence relations. The first one is the standard one which leads to the $2^{\text {nd }}$ cohomology group $H^{2}(G, U(1))$, where two two-cocycles are cohomologous if they differ by a coboundary. According to this, we associate with $\xi$ the class $[[\xi]]$, the elements of which differ in a coboundary generated by an arbitrary function on $G$. With this equivalence class we can associate a series of parameters, given by the corresponding element of $H^{2}(G, U(1))$, and will be called the cohomology parameters. An example of them is the mass parameter which characterizes the central extensions of the Galilei group. However, the previous considerations suggest that each equivalence class $[[\xi]]$ should be further partitioned according to what we have called pseudo-cohomology classes, $[\xi]$, the elements of which differ in a coboundary $\xi_{\alpha}$ generated by a function $\alpha$ on $G$ having trivial gradient at the identity. Pseudo-cohomology classes are then characterized by coadjoints orbits of $\mathcal{G}^{*}$ which satisfy the integrality condition [3] (the condition of integrality is associated with the globality of the generating function $\lambda$ on the group). 


\section{Group Approach to Quantization}

The original Group Approach to Quantization deals with a connected Lie group which is also a principal $U(1)$-bundle, either a central extension or not. The vertical subgroup, $U(1)$, simply realizes the well-established phase invariance in Quantum Mechanics through the $U(1)$-equivariance condition, on complex functions defined on $\tilde{G}$, intended to restrict this space of functions to the linear space of sections of an associated line bundle. The whole construction is defined in terms of canonical differential structures on the Lie group. However, the quantization formalism can be generalized in two directions. On the one hand, the structure group can be replaced by a larger, non-necessarily abelian group $T$ containing $U(1)$. In that case, operators in $T$, other than the standard $U(1)$, play the role of constraints as we generalize, accordingly, the equivariance condition [27. On the other hand, a non-connected group can substitute $\tilde{G}$. To proceed in this way we must replace all infinitesimal operations on the functions on the group by their finite counterparts. This generalization allows us to deal with groups with arbitrary homotopy and therefore to quantize systems the configuration space (or phase space) of which are not simply connected [28]. We shall be concerned here with $U(1)$-principal bundles on a connected base.

\subsection{U(1)-quantization}

Let us therefore consider a Lie group $\tilde{G}$ which is a $U(1)$-principal bundle with the bundle projection $\pi: \tilde{G} \rightarrow G$ being a group homomorphism. We denote by $\Theta$ the connection 1-form constructed as explained earlier. It satisfies $i_{\Xi} \Theta=1, L_{\Xi} \Theta=0, \Xi$ being the infinitesimal generator of $U(1)$, or the fundamental vector field of the principal bundle, which is in the centre of the Lie algebra $\tilde{\mathcal{G}} \equiv T_{e} \tilde{G}$. Since $\Theta$ is left-invariant it will be preserved $\left(L_{\tilde{X}^{R}} \Theta=\right.$ 0 ) by all right-invariant vector fields (generating finite left translations) on $\tilde{G}$. These vector fields are candidates to be infinitesimal generators of unitary transformations. To determine the space of functions on which they should act, we first select complex valued equivariant functions by requiring

$$
L_{\Xi} \psi=i \psi \text {. }
$$

In other terms, we start with $\Psi: \tilde{G} \rightarrow C$ and impose the equivariance condition (41) which identifies them as sections of an associated line bundle 
(see, for instance, [29]). To make the action of the right-invariant vector fields on them irreducible, we have to select appropriate subspaces, and this will be achieved by polarization conditions,

$$
L_{\tilde{X}^{L}} \psi=0, \forall \tilde{X}^{L} \in \mathcal{P},
$$

where the polarization subalgebra $\mathcal{P}$ is to be discussed at length.

The 2-form $\tilde{\Sigma} \equiv d \Theta$ is left invariant under $\tilde{G}$, and projectable onto a leftinvariant 2 -form $\Sigma$ of $G$. This, evaluated at the identity, defines a two-cocycle on the Lie algebra $\mathcal{G}$.

On vector fields $\mathcal{X}(G)$ we can define a "generalized Lagrange bracket" [30] by setting, for any pair of vector fields $X, Y \in \mathcal{X}(G)$,

$$
(X, Y)_{\Sigma}=\Sigma(X, Y) \in \mathcal{F}(G)
$$

In particular, when we consider left-invariant vector fields $X^{L}, Y^{L} \in$ $\mathcal{X}^{L}(G)$, we get a real valued bracket:

$$
\left(X^{L}, Y^{L}\right)_{\Sigma}=\Sigma\left(X^{L}, Y^{L}\right) \in R
$$

By evaluating $\tilde{\Sigma}$ at the identity of the group, i.e. on $T_{e} \tilde{G}=\tilde{\mathcal{G}}$, we can bring it to normal form, which would be the analog of a Darboux frame in the space of left-invariant 1 -forms. We can write

$$
\tilde{\Sigma}=\sum_{a=1}^{k} \theta^{L a} \wedge \theta^{L a+k}
$$

where $\theta^{L a}, \theta^{L a+k}, a=1, \ldots, k$ are left-invariant 1 -forms. We can define a (1,1)-tensor field $J$, a partial (almost) complex structure, by setting:

$$
\begin{aligned}
J \theta^{L a} & =\theta^{L a+k} \\
J \theta^{L a+k} & =-\theta^{L a} \\
J \theta^{L l} & =0
\end{aligned}
$$

where $\theta^{L l}$ are the remaining elements of a basis of left-invariant 1-forms not appearing in $\tilde{\Sigma}$ (that is, those related to $\operatorname{Ker} \tilde{\Sigma}$.). We also have a "partial metric tensor" $\rho$ by setting $\rho\left(\theta^{L a}, \theta^{L a^{\prime}}\right)=\delta_{a a^{\prime}}, \rho\left(\theta^{L a}, \theta^{L l}\right)=0, \rho\left(\theta^{L l}, \theta^{L l^{\prime}}\right)=$ 0 . 
Usually our considerations will be restricted to finite-dimensional Lie groups or infinite-dimensional ones possessing a countable basis of generators for which, for arbitrary fixed $\tilde{X}^{L}, \tilde{\Sigma}\left(\tilde{X}^{L}, \tilde{Y}^{L}\right)=0$ except for a finite number of vector fields $\tilde{Y}^{L}$ (finitely non-zero two-cocycle), and therefore this partial (almost) complex structure $J$ can always be introduced.

It is possible to associate with $\Theta$ an horizontal projector, a (1,1)-tensor field. We first define the vertical projector $V_{\Theta}(X)=\Theta(X) \Xi$, and then $H_{\Theta}=$ $I-V_{\Theta}$.

The characteristic module of $\Theta$ is defined as the intersection of $\operatorname{Ker} \Theta$ and $\operatorname{Kerd} \Theta=\operatorname{Ker} \tilde{\Sigma}$. It is generated by a subalgebra of $\mathcal{X}^{L}(\tilde{G})$, the characteristic subalgebra $\mathcal{G}_{C}$. Elements in $\operatorname{Ker} \Theta \cap \operatorname{Kerd} \Theta$ are easily shown to be a Lie algebra. In fact, it follows from the identity $d \Theta(X, Y)=L_{X} \Theta(Y)-$ $L_{Y} \Theta(X)-\Theta([X, Y])$.

It turns out that $P \equiv \tilde{G} / \mathcal{G}_{C}$ is a quantum manifold in the sense of Geometric Quantization, with connection the projection of $\Theta$ to $P$ (see [2]), and $d \Theta$ projected onto $P / U(1)$ is a symplectic 2 -form. This establishes the connection with the Coadjoint Orbits Method, the different coadjoint orbits being obtained by suitable choice of the (pseudo-)extension parameters.

A first-order polarization or just polarization $\mathcal{P}$ is defined as a maximal horizontal left subalgebra. The horizontality condition means that the polarization is in $\operatorname{Ker} \Theta$. Again, by using the identity $d \Theta(X, Y)=$ $L_{X} \Theta(Y)-L_{Y} \Theta(X)-\Theta([X, Y])$ we find that the generalized Lagrange braket of any two elements of $\mathcal{P}$ vanishes. Therefore we find that a polarization is an isotropic maximal subalgebra. We notice that maximality refers to the Lie commutator (subalgebra) and not to isotropy (Lagrange bracket).

A polarization may have non-trivial intersection with the characteristic subalgebra. We say that a polarization is full (or regular) if it contains the whole characteristic subalgebra. We also say that a polarization $\mathcal{P}$ is symplectic if $\tilde{\Sigma}$ on $\mathcal{P} \oplus J \mathcal{P}$ is of maximal rank [When the quantization 1 -form $\Theta$ is associated with a co-adjoint orbit of $G$, full polarizations satisfy Pukanszky's condition, and full and symplectic polarizations correspond to "admissible" subalgebras subordinated to $\left.\left.\Theta\right|_{e}[3]\right]$.

It should be stressed that the notion of polarization and characteristic subalgebras here given in terms of $\Theta$ is really a consequence of the fibre bundle structure of the group law of $\tilde{G}$ and, therefore, can be translated into finite (versus infinitesimal) form defining the corresponding subgroups (see [27]). 
From the geometric point of view, a polarization defines a foliation via the Frobenius theorem. It is possible to select subspaces of equivariant complexvalued functions on $\tilde{G}$, by requiring them to be constant along integral leaves of the foliation associated with the polarization. Whether this subspace is going to carry an irreducible representation for the right-invariant vector fields is to be checked. When the polarization is full and symplectic we get leaves which are maximally isotropic submanifolds for $d \Theta$. The selected subspaces of equivariant complex-valued functions on $\tilde{G}$, which we may call wave functions, will be characterized by $L_{\Xi} \Psi=i \Psi, L_{X^{L}} \Psi=0, \forall X^{L} \in \mathcal{P}$.

Finally, the Hilbert space structure on the space of wave functions is provided by the invariant Haar measure constituted by the exterior product of the components of the left-invariant canonical 1-form: $\mu=\theta^{L g^{1}} \wedge \theta^{L g^{2}} \wedge \ldots$. The finiteness of the scalar product will eventually restrict the values of the (pseudo-)extension parameters that characterize the representations.

The classical theory for the system is easily recovered by defining the Noether invariants as $F_{g^{i}} \equiv i_{\tilde{X}_{g^{i}}} \Theta$. A Poisson bracket can be introduced (defined by $d \Theta$ ), in such a way that the Noether invariants generate a Lie algebra isomorphic (if there are no algebraic anomalies, see Sec. 3) to that of $\tilde{G}$ (see [2] for a complete description of the classical theory using the GAQ formalism).

\subsection{Non-horizontal polarizations}

We can generalize the notion of polarization introduced above by relaxing the condition of horizontality, and define a non-horizontal polarization as a maximal left subalgebra of $\tilde{\mathcal{G}}^{L}$ not containing the vertical generator $\Xi$. Although this seems to be a more general notion, the following proposition states that it is related to the previous one.

Proposition: Given a Quantization 1-form $\Theta$ and a non-horizontal polarization $\mathcal{P}$ with respect to it, it is always possible to find a left-invariant 1-form $\Theta^{\prime}=\Theta+\alpha_{i} \theta^{L g^{i}}$ for which $\mathcal{P}$ is horizontal.

Proof: Let $\left\{X_{(k)}^{L}\right\}_{k=1}^{m}$ be a basis for $\mathcal{P}$, where $m=\operatorname{dim} \mathcal{P}$, and denote $a_{(k)}^{0} \equiv \Theta\left(X_{(k)}^{L}\right)$, which are, in general, non-zero for strictly non-horizontal polarizations. The condition for $\mathcal{P}$ of being horizontal with respect to $\Theta^{\prime}$ is 
written as:

$$
\sum_{i=1}^{n} \alpha_{i} a_{(k)}^{i}=-a_{(k)}^{0}, \quad k=1, \ldots, m,
$$

where $a_{(k)}^{i} \equiv \theta^{L g^{i}}\left(X_{(k)}^{L}\right)$, and $n=\operatorname{dim} \mathcal{G}$. This is an undetermined equation for the $\alpha_{i}, i=1, \ldots, n$, and the dimension of the space of solutions, since $X_{(k)}^{L}$ form a basis for $\mathcal{P}$, is $\operatorname{dim} \mathcal{G}-\operatorname{dim} \mathcal{P}$.

Note that, up to an (irrelevant) total differential, $\Theta^{\prime}$ coincides with the quantization 1-form obtained after adding a coboundary generated by a function $\lambda$ with $\lambda_{i}^{0}=\alpha_{i}$. Therefore, polarizing with a non-horizontal polarization is equivalent to introducing a pseudo-cocycle in the group law (and polarize with respect to a horizontal polarization), and, accordingly, non-horizontal polarizations can be classified according to pseudo-cohomology classes. This equivalence will be exploited in some of the examples. In addition, in generalizing first-order polarizations to higher-order ones, the notion of nonhorizontal polarization will turn out to be a more appropiate one to start with.

\section{Simple Examples}

\subsection{The abelian group $R^{k}$}

The simplest example one can think of is the abelian group $R^{k}$, with $k>1$ (the case $k=1$ is trivial, since it admits no non-trivial symplectic structure). Since the coadjoint action is trivial, all its coadjoint orbits are zero dimensional. This means that there will be no pseudo-cohomology classes, and only true central extensions have to be considered.

Given any two-cocycle $\xi$ defining a central extension $\tilde{R}^{k}$ of $R^{k}$, then $d \Theta$, where $\Theta$ is the quantization 1-form, is always left- (and right- in this case) invariant and exact, but it is not invariantly exact (as a 2-form on $R^{k}$ ), due to the non-trivial group cohomology of $R^{k}$. If we parametrize $R^{k}$ with (global) canonical coordinates $\vec{x}=\left(x_{1}, x_{2}, \ldots, x_{k}\right)$, then, since it is left invariant and the group is abelian, $d \Theta$ can be written as:

$$
d \Theta=a_{i j} d x^{i} \wedge d x^{j}
$$

where $a_{i j}$ is an antisymmetric $k \times k$ constant matrix. We can choose a two- 
cocycle representative of the associated cohomology class of the form:

$$
\xi\left(\vec{x}_{1}, \vec{x}_{2}\right)=a_{i j} x_{1}^{i} x_{2}^{j},
$$

any other representative will differ from this by a coboundary that, due to the trivial pseudo-cohomology of $R^{k}$, will contribute to $\Theta$ with an irrelevant total differential. Therefore, $\xi$ is an anti-symmetric bilinear function on $R^{k}$, and with an appropriate change of coordinates in $R^{k}$ can be taken to normal form, in which the matrix $a_{i j}$ is written as:

$$
\frac{1}{2}\left(\begin{array}{c|c|c}
\frac{0_{n}}{-D_{n}} \mid \frac{D_{n}}{0_{n}} & 0_{2 n \times r} \\
0_{r \times 2 n} & 0_{r}
\end{array}\right),
$$

where $0_{p}$ is the $p \times p$ zero matrix, $0_{p \times q}$ is the zero $p \times q$ matrix, and $D_{n}$ is a $n \times n$ real matrix of the form:

$$
\left(\begin{array}{cccccc}
\nu_{1} & 0 & \cdot & \cdot & \cdot & 0 \\
0 & \nu_{2} & \cdot & \cdot & \cdot & 0 \\
\cdot & \cdot & \cdot & \cdot & \cdot & \cdot \\
\cdot & \cdot & \cdot & \cdot & \cdot & \cdot \\
\cdot & \cdot & \cdot & \cdot & \nu_{n-1} & 0 \\
0 & \cdot & \cdot & \cdot & 0 & \nu_{n}
\end{array}\right)
$$

with $k=2 n+r$. The parameters $\nu_{1}, \ldots, \nu_{n}$ characterize the extension $\tilde{R}^{k}$, and, thus, they are the cohomology parameters. In physical situations, the subspace $R^{2 n}$ of $R^{k}$ is the tangent space $T\left(R^{n}\right)$ of a physical system (at this point there is no distinction between the tangent or the phase space $T^{*}\left(R^{n}\right)$, and we shall consider it the tangent space for convenience). The requirement of isotropy (under spatial rotations) will fix these parameters to coincide, $\nu_{i}=\nu, \forall i=1, \ldots, n$. In this case, the two-cocycle can be written as:

$$
\xi\left(\vec{q}_{1}, \vec{v}_{1}, \vec{a}_{1} ; \vec{q}_{2}, \vec{v}_{2}, \vec{a}_{2}\right)=\frac{1}{2} \nu\left(\vec{q}_{2} \cdot \vec{v}_{1}-\vec{q}_{1} \cdot \vec{v}_{2}\right),
$$

where $\vec{q}_{1,2}$ are $n$-dimensional vectors corresponding to the first $n$ coordinates (in the new basis), $\vec{v}_{1,2}$ correspond to the following $n$ "conjugated" coordinates, and $\vec{a}_{1,2}$ to the remaining $r$ coordinates. Since the two-cocycle $\xi$ 
does not depend on the coordinates $\vec{a}_{1,2}$, the group $\tilde{R}^{k}$ can be written as $\mathrm{H}-\mathrm{W}_{n} \times R^{r}$, where $\mathrm{H}-\mathrm{W}_{n}$ is the well-known Heisenberg-Weyl group with $n$ coordinates and $n$ velocities. The group law for $\tilde{R}^{k}$ can be rewritten in terms of the new coordinates:

$$
\begin{aligned}
\vec{q}^{\prime \prime} & =\vec{q}^{\prime}+\vec{q} \\
\vec{v}^{\prime \prime} & =\vec{v}^{\prime}+\vec{v} \\
\vec{a}^{\prime \prime} & =\vec{a}^{\prime}+\vec{a} \\
\zeta^{\prime \prime} & =\zeta^{\prime} \zeta e^{\frac{i}{2} \nu\left(\vec{q}^{\prime} \vec{v}-\vec{v}^{\prime} \vec{q}\right)} .
\end{aligned}
$$

From the group law we see that if $\vec{q}$ is interpreted as coordinates, and $\vec{v}$ as velocities, then $\nu=\frac{m}{\hbar}$. Therefore, the cohomology parameter for the (isotropic) Heisenberg-Weyl group can be identified with $\frac{m}{\hbar}$. The variables $\vec{a}$ do not play any role, and can be factorized, as we shall see later.

Left- and right-invariant (under the group $\tilde{R}^{k}$ ) vector fields are:

$$
\begin{aligned}
\tilde{X}_{\vec{q}}^{L} & =\frac{\partial}{\partial \vec{q}}-\frac{m}{2 \hbar} \vec{v} \Xi & \tilde{X}_{\vec{q}}^{R} & =\frac{\partial}{\partial \vec{q}}+\frac{m}{2 \hbar} \overrightarrow{v \Xi} \\
\tilde{X}_{\vec{v}}^{L} & =\frac{\partial}{\partial \vec{v}}+\frac{m}{2 \hbar} \vec{q} \Xi & \tilde{X}_{\vec{v}}^{R} & =\frac{\partial}{\partial \vec{v}}-\frac{m}{2 \hbar} \vec{q} \Xi \\
\tilde{X}_{\vec{a}}^{L} & =\frac{\partial}{\partial \vec{a}} & \tilde{X}_{\vec{a}}^{R} & =\frac{\partial}{\partial \vec{a}},
\end{aligned}
$$

and $\Xi=i \zeta \frac{\partial}{\partial \zeta}$ is the vertical (left- and right-invariant) vector field. The commutation relations for these vector fields are:

$$
\left[\tilde{X}_{q^{i}}^{L}, \tilde{X}_{v^{j}}^{L}\right]=\frac{m}{\hbar} \Xi
$$

the rest of them being zero. Left and right invariant 1-forms for $R^{k}$ are simply $d \vec{x}$, for $\vec{x}=\vec{q}, \vec{v}$ and $\vec{a}$. The quantization 1-form $\Theta$, which for convenience we redefine with a factor $\hbar$, is:

$$
\Theta=\hbar \frac{d \zeta}{i \zeta}+\frac{1}{2}(m \vec{v} \cdot d \vec{q}-\vec{q} \cdot d(m \vec{v})) .
$$

Note that $d \Theta=d(m \vec{v}) \wedge d \vec{q}$ is a pre-symplectic form on $R^{k}$, with kernel the subspace $R^{r}$ spanned by the vectors $\vec{a}$. On the quotient $R^{k} / R^{r}=R^{2 n}$, $d \Theta$ is a true symplectic form. In fact, a partial complex structure $J$ can be introduced, of the form $J=d\left(m v^{i}\right) \otimes \tilde{X}_{q^{i}}^{L}-\frac{1}{m} d q^{i} \otimes \tilde{X}_{v^{i}}^{L}$ (that is, $J$ satisfies $J\left(\tilde{X}_{q^{i}}^{L}\right)=-\frac{1}{m} \tilde{X}_{v^{i}}^{L}$ and $\left.J\left(\tilde{X}_{v i}^{L}\right)=m \tilde{X}_{q^{i}}^{L}\right) . J$ turns out to be a complex 
structure on the reduced space $R^{k} / R^{r}$. The characteristic subalgebra, i.e. $\operatorname{Ker} \Theta \cap \operatorname{Kerd} \Theta$, is accordingly given by $\mathcal{G}_{\Theta}=<\tilde{X}_{\vec{a}}^{L}>$.

The possible horizontal polarizations for this group are of the form:

$$
\mathcal{P}=<\tilde{X}_{\vec{a}}^{L}, \alpha_{i}^{j} \tilde{X}_{q^{j}}^{L}+\beta_{i}^{j} \tilde{X}_{v_{j}}^{L}, i=1, \ldots, n>,
$$

with restrictions on the real coefficients $\alpha_{i}^{j}, \beta_{i}^{j}$ making it maximal, and horizontal with respect to $\Theta$. These restrictions are necessary for the polarizations to be full and symplectic. If, again, one imposes isotropy under rotations (if rotations are taken into account they must be included in the polarization), the coefficients $\alpha_{i}^{j}$ and $\beta_{i}^{j}$ must be proportional.

There are two "natural" polarizations, $\mathcal{P}_{p}=<\tilde{X}_{\vec{a}}^{L}, \tilde{X}_{\vec{q}}^{L}>$ and $\mathcal{P}_{q}=<$ $\tilde{X}_{\vec{a}}^{L}, \tilde{X}_{\vec{v}}^{L}>$, leading to the representations in momentum and configuration space, respectively. It should be stressed that these polarizations lead to equivalent representations of $R^{k}$, and the unitary operator relating the representations obtained with $\mathcal{P}_{p}$ and $\mathcal{P}_{q}$ is the Fourier transform. This is an outer isomorphism of $\mathrm{H}-\mathrm{W}_{n}$, but it is inner in the Weyl-Symplectic group $W S p(2 n, R)$ [31], as we shall see later.

Taking advantage of the natural complex structure of $R^{2 n} \approx C^{n}$ (the one induced by $J$ ), we can choose a complex polarization of the form:

$$
\mathcal{P}_{c}=<\tilde{X}_{\vec{a}}^{L}, \tilde{X}_{\vec{q}}^{L}+i \omega \tilde{X}_{\vec{v}}^{L}>,
$$

where $\omega$ is a constant with the appropriate dimensions (from the physical point of view, it will be frequency, which makes this polarization appropriate for the description of the Harmonic Oscillator; see Sec. 5.2). This polarization leads to a representation in terms of holomorphic (or anti-holomorphic) functions on $C^{n}$. It is unitarily equivalent to the other representations, the unitary transformation which relates it with the representation in configuration space being the Bargmann transform. This is also an outer automorphism of $\mathrm{H}-\mathrm{W}_{n}$, but it is inner in a certain subsemigroup of $S p(2 n, C)$ [31.

Let us compute the representation obtained with the polarization $\mathcal{P}_{q}$. The equations $\tilde{X}_{\vec{a}}^{L} \Psi=0$ leads to wave functions not depending on the $\vec{a}$ variables (they trivially factorize and we can forget about them), and the equations $\tilde{X}_{\vec{v}}^{L} \Psi=0$ (together with the equivariance condition $\Xi \Psi=i \Psi$ ) lead to:

$$
\Psi=\zeta e^{-\frac{i m}{2 \hbar} \vec{q} \cdot \vec{v}} \Phi(\vec{q}),
$$


where $\Phi(\vec{q})$ is an arbitrary function of $\vec{q}$ (appart from normalizability considerations). If we compute the action of the right-invariant vector fields on these wave functions, we obtain:

$$
\begin{aligned}
& \tilde{X}_{\vec{q}}^{R} \Psi=\zeta e^{-\frac{i m}{2 \hbar} \vec{q} \cdot \vec{v}} \frac{\partial}{\partial \vec{q}} \Phi(\vec{q}) \\
& \tilde{X}_{\vec{v}}^{R} \Psi=-\zeta e^{-\frac{i m}{2 \hbar} \vec{q} \cdot \vec{v}} \frac{i m}{\hbar} \vec{q} \Phi(\vec{q}) .
\end{aligned}
$$

This representation is unitarily equivalent to the Schrödinger representation (labeled by the values of the cohomology parameter $\frac{m}{\hbar}$ ) of the $\mathrm{H}-\mathrm{W}$ group, and the unitary operator relating them is the operator $e^{-\frac{i m}{2 \hbar} \vec{q} \cdot \vec{v}}$. As expected, it reproduces the standard Weyl commutation relations, the basis of nonrelativistic Quantum Mechanics.

With the polarization $\mathcal{P}_{v}^{H O}$ we obtain the representation in velocity (momentum) space, with wave functions of the form:

$$
\Psi=\zeta e^{\frac{i m}{2 \hbar} \vec{q} \cdot \vec{v}} \Phi(\vec{v}),
$$

where $\Phi(\vec{v})$ is an arbitrary function of $\vec{v}$. The action of the right-invariant vector fields are:

$$
\begin{aligned}
& \tilde{X}_{\vec{q}}^{R} \Psi=\zeta e^{\frac{i m}{2 \hbar} \vec{q} \cdot \vec{v}}\left(\frac{i m}{\hbar} \vec{v}\right) \Phi(\vec{v}) \\
& \tilde{X}_{\vec{v}}^{R} \Psi=\zeta e^{\frac{i m}{2 \hbar} \vec{q} \cdot \vec{v}}\left(\frac{\partial}{\partial \vec{v}}\right) \Phi(\vec{v}) .
\end{aligned}
$$

If we now solve for the holomorphic polarization $\mathcal{P}_{c}$, we obtain, introducing the appropriate (complex) change of variables $\vec{c} \equiv \frac{1}{\sqrt{2}}(\vec{q}+i \vec{v} / \omega)$ and its complex conjugate, the following wave functions:

$$
\Psi=e^{\frac{m \omega}{2 \hbar} \vec{c} \cdot \vec{c}^{*}} \Phi(\vec{c}),
$$

where $\Phi(\vec{c})$ is an arbitrary holomorphic function. The action of the rightinvariant vector fields is:

$$
\begin{aligned}
& \tilde{X}_{\vec{c}}^{R} \Psi=e^{\frac{m \omega}{2 \hbar} \vec{c} \cdot \vec{c}^{*}}\left(\frac{m \omega}{\hbar} \vec{c}\right) \Phi(\vec{c}) \\
& \tilde{X}_{\vec{c}^{*}}^{R} \Psi=e^{\frac{m \omega}{2 \hbar} \vec{c} \cdot \vec{c}^{*}}\left(\frac{\partial}{\partial \vec{c}}\right) \Phi(\vec{c}),
\end{aligned}
$$


leading to the standard Bargmann's holomorphic representation. The generators $\tilde{X}_{\vec{c}}^{R}$ and its complex conjugate have the usual form $\frac{1}{\sqrt{2}}\left(\tilde{X}_{\vec{q}}^{R} \mp \omega \tilde{X}_{\vec{v}}^{R}\right)$.

Finally, we shall consider another real polarization, of the form $<\tilde{X}_{\vec{a}}^{L}, \tilde{X}_{\vec{q}}^{L}+$ $\omega \tilde{X}_{\vec{v}}^{L}>$. Introducing the adequate change of variables, $\vec{\rho}_{ \pm} \equiv \frac{1}{\sqrt{2}}(\vec{q} \pm \vec{v} / \omega)$, the polarization equations lead to the wave functions:

$$
\Psi=e^{-i \frac{m \omega}{2 \hbar} \vec{\rho}_{+} \cdot \vec{\rho}_{-}} \Phi\left(\vec{\rho}_{-}\right),
$$

where $\Phi\left(\vec{\rho}_{-}\right)$is an arbitrary function of $\vec{\rho}_{-}$. As we shall see later, this representation is adequate for the description of the repulsive Harmonic Oscillator, see Sec. 5.2. The action of the right-invariant vector fields are:

$$
\begin{aligned}
& \tilde{X}_{\vec{\rho}_{+}}^{R} \Psi=-e^{-i \frac{m \omega}{2 \hbar} \vec{\rho}_{+} \cdot \vec{\rho}_{-}}\left(\frac{i m \omega}{\hbar} \vec{\rho}_{-}\right) \Phi\left(\vec{\rho}_{-}\right) \\
& \tilde{X}_{\vec{\rho}_{-}}^{R} \Psi=e^{-i \frac{m \omega}{2 \hbar} \vec{\rho}_{+} \cdot \vec{\rho}_{-}}\left(\frac{\partial}{\partial \vec{\rho}_{-}}\right) \Phi\left(\vec{\rho}_{-}\right)
\end{aligned}
$$

where $\tilde{X}_{\vec{\rho}_{ \pm}}^{R} \equiv \frac{1}{\sqrt{2}}\left(\tilde{X}_{\vec{q}}^{R} \pm \omega \tilde{X}_{v}^{R}\right)$.

In this simple example no temporal evolution is considered. This question will be addressed in Sec. 5.2, with the example of the Schrödinger group, the group of linear canonical transformation acting on the H-W group. Different choices of "time" in it (among different uniparametric subgroups of $S L(2, R)$ ) will provide us with different dynamical systems, like the free particle, the harmonic oscillator and the repulsive oscillator (see [31]). Each time generator will select, among the ones considered here, the appropriate (invariant) polarization.

\subsection{The semisimple group $S U(2)$}

Let us consider now an example, which in a certain sense is on the other extreme to that of the abelian group $R^{k}$. It is the semisimple group $S U(2)$, which has trivial cohomology group, $H^{2}(S U(2), U(1))=0$. For this reason all two-cocycles on $S U(2)$ are coboundaries, and they will be classified according to pseudo-cohomology classes only.

A group law for $S U(2)$ can be obtained making use of its realization as $2 \times 2$ complex matrices of the form:

$$
\left(\begin{array}{cc}
z_{1} & z_{2} \\
-z_{2}^{*} & z_{1}^{*}
\end{array}\right)
$$


with $\left|z_{1}\right|^{2}+\left|z_{2}\right|^{2}=1$, with matrix multiplication as group law.

We shall proceed in an indirect way in order to keep global coordinates. Let us relax the condition $\left|z_{1}\right|^{2}+\left|z_{2}\right|^{2}=1$ to $\left|z_{1}\right|^{2}+\left|z_{2}\right|^{2}>0$, and apply the formalism to this group.

The group law, obtained from the multiplication of matrices, is:

$$
\begin{aligned}
z_{1}^{\prime \prime} & =z_{1}^{\prime} z_{1}-z_{2}^{\prime} z_{2}^{*} \\
z_{2}^{\prime \prime} & =z_{1}^{\prime} z_{2}+z_{2}^{\prime} z_{1}^{*} \\
z_{1}^{* \prime \prime} & =\left(z_{1}^{\prime \prime}\right)^{*} \\
z_{2}^{* \prime \prime} & =\left(z_{2}^{\prime \prime}\right)^{*} .
\end{aligned}
$$

Left- and right-invariant vector fields are particularly simple:

$$
\begin{aligned}
X_{z_{1}}^{L} & =z_{1} \frac{\partial}{\partial z_{1}}+z_{2}^{*} \frac{\partial}{\partial z_{2}^{*}} & X_{z_{1}}^{R} & =z_{1} \frac{\partial}{\partial z_{1}}+z_{2} \frac{\partial}{\partial z_{2}} \\
X_{z_{2}}^{L} & =z_{1} \frac{\partial}{\partial z_{2}}+z_{2}^{*} \frac{\partial}{\partial z_{1}^{*}} & X_{z_{1}}^{R} & =z_{1}^{*} \frac{\partial}{\partial z_{2}}-z_{2}^{*} \frac{\partial}{\partial z_{1}} \\
X_{z_{1}^{*}}^{L} & =z_{1}^{*} \frac{\partial}{\partial z_{1}^{*}}+z_{2} \frac{\partial}{\partial z_{2}} & X_{z_{1}^{*}}^{R} & =z_{1}^{*} \frac{\partial}{\partial z_{1}^{*}}+z_{2}^{*} \frac{\partial}{\partial z_{2}^{*}} \\
X_{z_{2}^{*}}^{L} & =z_{1}^{*} \frac{\partial}{\partial z_{2}^{*}}+z_{2} \frac{\partial}{\partial z_{1}} & X_{z_{2}^{*}}^{R} & =z_{1} \frac{\partial}{\partial z_{2}^{*}}-z_{2} \frac{\partial}{\partial z_{1}^{*}}
\end{aligned}
$$

and the commutation relations for left-invariant vector fields are:

$$
\begin{aligned}
{\left[X_{z_{1}}^{L}, X_{z_{2}}^{L}\right] } & =X_{z_{2}}^{L} & {\left[X_{z_{1}^{*}}^{L}, X_{z_{2}}^{L}\right] } & =-X_{z_{2}}^{L} \\
X_{z_{1}}^{L}, X_{z_{1}^{*}}^{L} & =0 & \left.X_{z_{1}^{*}}^{L}, X_{z_{2}^{*}}^{L}\right] & =X_{z_{2}^{*}}^{L} \\
X_{z_{1}}^{L}, X_{z_{2}^{*}}^{L} & =-X_{z_{2}^{*}}^{L} & {\left[X_{z_{2}}^{L}, X_{z_{2}^{*}}^{L}\right] } & =-X_{z_{1}}^{L}+X_{z_{1}^{*}}^{L},
\end{aligned}
$$

Note that the combination $X_{z_{1}}^{L}+X_{z_{1}^{*}}^{L}$ is a central generator, commuting with all the other vector fields. It is a gauge [32] generator, and it will be contained in any polarization. Therefore its action (since it is central, it is left- and right-invariant) on the wave functions is always trivial. Thus, our Lie algebra decomposes in the direct sum $s u(2) \oplus R$, where $s u(2)$ is generated by $X_{z_{1}}^{L}-X_{z_{1}^{*}}^{L}, X_{z_{2}}^{L}$ and $X_{z_{2}^{*}}^{L}$. In this way we return to our original problem of quantizing $S U(2)$, but with the advantage of using the global coordinates $z_{1}$ and $z_{2}$, which will allow us to use finite transformations (not reachable with local coordinates ) to implement the compatibility of charts.

Now we should consider central extensions of $S U(2)$, which, due to its trivial group cohomology, will be pseudo-extesions, classified according to pseudo-cohomology classes. However, in order to illustrate the equivalence 
between pseudo-extensions and non-horizontal polarizations, we shall consider the direct product $S U(2) \times U(1)$ (trivial pseudo-extension) and introduce an appropriate non-horizontal polarization.

In this case the group law for the variable $\zeta \in U(1)$ is simply $\zeta^{\prime \prime}=\zeta^{\prime} \zeta$ and the generator associated with it, which is both left- and right-invariant, is $\Xi=i \zeta \frac{\partial}{\partial \zeta}$. The quantization 1 -form is $\Theta=\frac{d \zeta}{i \zeta}$. Since the extension is trivial, the whole group $S U(2)$ (times $R$, generated by the gauge generator $\left.X_{z_{1}}^{L}+X_{z_{1}^{*}}^{L}\right)$ has its infinitesimal generators in the caracteristic subalgebra. A horizontal polarization would be constituted by the caracteristic subalbegra, and it would lead, obviously, to the trivial representation of $S U(2)$, with spin zero.

Let us introduce, instead, a non horizontal polarization of the form:

$$
\mathcal{P}^{n . h .}=<X_{z_{1}}^{L}+X_{z_{1}^{*}}^{L}, X_{z_{1}}^{L}-X_{z_{1}^{*}}^{L}-\lambda \Xi, X_{z_{2}}^{L}>,
$$

where $\lambda \in R$ is a parameter characterizing the polarization. Later, we shall see that the integrability of the polarization equations to the whole group will restrict the possibles values of $\lambda$. Solving the first polarization equation, $\left(X_{z_{1}}^{L}+X_{z_{1}^{*}}^{L}\right) \Psi=0$, which states the gauge caracter of this generator, leads to a wave function defined on $S U(2)$,

$$
\Psi=\Psi\left(w_{1}, w_{2}, w_{1}^{*}, w_{2}^{*}\right)
$$

where $w_{i}=\frac{z_{i}}{\sqrt{\left|z_{1}\right|^{2}+\left|z_{2}\right|^{2}}}, i=1,2$, in such a way that $\left|w_{1}\right|^{2}+\left|w_{2}\right|^{2}=1$, as expected.

The second polarization equation (together with the $U(1)$-equivariance condition $\Xi \Psi=i \Psi)$, written in terms of the new variables, reads:

$$
\left[w_{1} \frac{\partial}{\partial w_{1}}+w_{2}^{*} \frac{\partial}{\partial w_{2}^{*}}-w_{1}^{*} \frac{\partial}{\partial w_{1}^{*}}-w_{2} \frac{\partial}{\partial w_{2}}-\lambda\right] \Psi=0,
$$

while the last polarization condition is written as:

$$
\left[w_{1} \frac{\partial}{\partial w_{2}}-w_{2}^{*} \frac{\partial}{\partial w_{1}^{*}}\right] \Psi=0 .
$$

At this point it is convenient to introduce local charts in $S U(2)$ in order to solve these polarization equations. Thus, two cases are considered: 
- $z_{1} \neq 0$ : The solutions are of the form:

$$
\Psi=w_{1}^{\lambda} \Phi(\tau)
$$

with $\Phi$ is an arbitrary holomorphic function of the variable $\tau \equiv \frac{w_{2}^{*}}{w_{1}}$.

- $z_{2}^{*} \neq 0$ : The solutions are of the form:

$$
\tilde{\Psi}=w_{2}^{* \lambda} \tilde{\Phi}(\tilde{\tau})
$$

where $\tilde{\Phi}$ is an arbitrary holomorphic function of the variable $\tilde{\tau} \equiv \frac{w_{1}}{w_{2}^{*}}=$ $\frac{1}{\tau}$

These solutions are the same that those one obtains considering stereographic projection coordinates on the sphere $S^{2} \approx S U(2) / U(1)$, but with the advantage that one can pass from one chart to the other with the action of the element $J=\left(\begin{array}{cc}0 & 1 \\ -1 & 0\end{array}\right) \in S U(2)$, which satisfies $J^{4}=I_{2}$. However, the repeated action of this element on the wave functions is $\Psi\left(J^{4} * g\right)=$ $(-1)^{-2 \lambda} \Psi(g)$, and, therefore, the requirement of single-valuedness of the representation (since the group $S U(2)$ is simply connected all its representations are single-valued) implies that $\lambda \in Z$. This condition is equivalent to the condition of chart compatibility.

Now we have to compute the action of the right-invariant vector fields on polarized wave functions. Since we can pass from one chart to the other with the aid of the element $J$, we only have to compute it on the chart containing the identity of the group:

$$
\begin{aligned}
\left(\tilde{X}_{z_{1}}^{R}-\tilde{X}_{z_{1}^{*}}^{R}\right) \Psi & =w_{1}^{\lambda}\left[\lambda \Phi-2 \tau \frac{\partial \Phi}{\partial \tau}\right] \\
\tilde{X}_{z_{2}}^{R} \Psi & =w_{1}^{\lambda}\left[-\lambda \tau+\tau^{2} \frac{\partial \Phi}{\partial \tau}\right] \\
\tilde{X}_{z_{2}^{*}}^{R} \Psi & =w_{1}^{\lambda}\left[\frac{\partial \Phi}{\partial \tau}\right] .
\end{aligned}
$$

It is straightforward to check that $w_{1}^{\lambda}$ and $w_{1}^{\lambda} \tau^{\lambda}$ are maximal and minimal weight states, respectively. Therefore the Hilbert space (on which the rightinvariant vector fields act irreducibily) has dimensinon $\lambda+1$ and is generated 
by the wave functions $\left\{w_{1}^{\lambda}, w_{1}^{\lambda} \tau, \ldots, w_{1}^{\lambda} \tau^{\lambda}\right\}$. Clearly, we can identify $\lambda$ with $2 j, j$ being the spin, which characterizes the irreducible representations of $S U(2)$.

\section{Algebraic Anomalies}

In Sec. 3, we introduced the concept of full and symplectic polarization subalgebra intended to reduce the representation obtained through the rightinvariant vector fields acting on equivariant functions on the group. It contains "half" of the symplectic vector fields as well as the entire characteristic subalgebra. If the full reduction is achieved, the whole set of physical operators can be rewritten in terms of the basic ones, i.e. those which are the right version of the left-invariant generators in $J \mathcal{P} \oplus J^{2} \mathcal{P}$. For instance, the energy operator for the free particle can be written as $\frac{\hat{p}^{2}}{2 m}$, the angular momentum in $3+1$ dimensions is the vector product $\hat{\mathbf{x}} \times \hat{\mathbf{p}}$, or the energy for the harmonic oscillator is $\hat{c}^{\dagger} \hat{c}$ (note that, since we are using first-order polarizations, all this operators are really written as first-order differential operators).

However, the existence of a full and symplectic polarization is guaranteed only for semisimple and solvable groups [3]. We define an anomalous group [25] as a central extension $\tilde{G}$ which does not admit any polarization which is full and symplectic for some values of the (pseudo-)cohomology parameters, called the classical values of the anomaly (they are called classical because they are associated with the coadjoints orbits of the group $\tilde{G}$, that is, with the classical phase space of the physical system). Anomalous groups feature another set of values of the (pseudo-)cohomology parameters, called the quantum values of the anomaly, for which the carrier space associated with a full and symplectic polarization contains an invariant subspace. For the classical values of the anomaly, the classical solution manifold undergoes a reduction in dimension thus increasing the number of (non-linear) relationships among Noether invariants, whereas for the quantum values the number of basic operators decreases on the invariant (reduced) subspace due to the appearance of (higher-order) relations among the quantum operators.

We must remark that the anomalies we are dealing with in this paper are of algebraic character in the sense that they appear at the Lie algebra level, and must be distinguished from the topologic anomalies which are associated with the non-trivial homotopy of the (reduced) phase space [28]. 
The non-existence of a full and/or symplectic polarization is traced back to the presence in the characteristic subalgebra, for certain values of the (pseudo-)cohomology parameters (the classical values of the anomaly), of some elements the adjoint action of which are not diagonalizable in the " $x-p$ like" algebra subspace. The anomaly problem here presented parallels that of the non-existence of invariant polarizations in the Kirillov-Kostant co-adjoint orbits method [33], and the conventional anomaly problem in Quantum Field Theory which manifests itself through the appearance of central charges in the quantum current algebra, absent from the classical (Poisson bracket) algebra [34].

The full reduction of representations in anomalous cases will be achieved by means of a generalized concept of (higher-order) polarization (see below).

Let us try to clarify the situation in terms of coordinates and employing a rather symbolic language. We may imagine our group $\tilde{G}$ parametrized by $\left(\kappa, \pi ; \epsilon^{+}, \epsilon^{-}, \epsilon^{0} ; \zeta\right)$ and with Lie algebra of the form:

$$
\begin{aligned}
{\left[\tilde{X}_{\kappa}^{L}, \tilde{X}_{\pi}^{L}\right] } & =\tilde{X}_{\epsilon^{0}}^{L}+a \Xi, a \in R \\
{\left[\tilde{X}_{\epsilon^{+}}^{L}, \tilde{X}_{\epsilon^{-}}^{L}\right] } & =\tilde{X}_{\epsilon^{0}}^{L} \\
{\left[\tilde{X}_{\epsilon^{ \pm}}^{L}, \tilde{X}_{\kappa}^{L}\right] } & =\alpha^{ \pm} \tilde{X}_{\kappa}^{L}+\beta^{ \pm} \tilde{X}_{\pi}^{L} \\
{\left[\tilde{X}_{\epsilon^{ \pm}}^{L}, \tilde{X}_{\pi}^{L}\right] } & =\gamma^{ \pm} \tilde{X}_{\kappa}^{L}+\delta^{ \pm} \tilde{X}_{p i}^{L},
\end{aligned}
$$

where it is assumed that the adjoint action of none of $\tilde{X}_{\epsilon^{ \pm}}^{L}$ is diagonalizable (only the action of $\tilde{X}_{\epsilon^{0}}^{L}$, here omitted, can be diagonalized). A glance at (78) reveals the structure of $\mathcal{G}_{C}$ :

$$
\mathcal{G}_{C}=<\tilde{X}_{\epsilon^{0}}^{L}, \tilde{X}_{\epsilon^{+}}^{L}, \tilde{X}_{\epsilon^{-}}^{L}>,
$$

and the fact that there is no full and symplectic polarization; only non-full (though symplectic) polarizations $\mathcal{P}^{ \pm}$are found which exclude $\tilde{X}_{\epsilon^{\mp}}^{L} \in \mathcal{G}_{C}$, and the non-symplectic (though full) polarization $\mathcal{G}_{C}$ itself. Quantizing with $\mathcal{P}^{ \pm}$means that $\tilde{X}_{\epsilon^{-}}^{R}$ and $\tilde{X}_{\epsilon^{+}}^{R}$ cannot be expressed in terms of $\tilde{X}_{\kappa}^{R}, \tilde{X}_{\pi}^{R}$. The situation is as if $\tilde{X}_{\epsilon \mp}^{R}$ also were basic operators, i.e. as if

$$
\left[\tilde{X}_{\epsilon^{+}}^{L}, \tilde{X}_{\epsilon^{-}}^{L}\right]=\tilde{X}_{\epsilon^{0}}^{L}+k \Xi
$$

for some numerical constant $k$. Quantizing with the polarization $\mathcal{P}_{C} \equiv \mathcal{G}_{C}$ leads to a rather unconventional representation in terms of the $\kappa$ 's and $\pi$ 's 
variables, like Van Hove's prequantization of quadratic polynomials on phasespace, which is reducible, decomposing in two invariant subspaces [1].

The reasoning above suggests another way of looking at anomalies. Let us start from a doubly extended group $\tilde{G}$ with initially independent (pseudo-) extension parameters $a, k$. This involves adopting the following Lie algebra:

$$
\begin{aligned}
{\left[\tilde{X}_{\kappa}^{L}, \tilde{X}_{\pi}^{L}\right] } & =\tilde{X}_{\epsilon^{0}}^{L}+a \Xi, \quad a \in R \\
{\left[\tilde{X}_{\epsilon^{+}}^{L}, \tilde{X}_{\epsilon^{-}}^{L}\right] } & =\tilde{X}_{\epsilon^{0}}^{L}+k \Xi, \quad k \in R \\
{\left[\tilde{X}_{\epsilon^{ \pm}}^{L}, \tilde{X}_{\kappa}^{L}\right] } & =\alpha^{ \pm} \tilde{X}_{\kappa}^{L}+\beta^{ \pm} \tilde{X}_{\pi}^{L} \\
{\left[\tilde{X}_{\epsilon^{ \pm}}^{L}, \tilde{X}_{\pi}^{L}\right] } & =\gamma^{ \pm} \tilde{X}_{\kappa}^{L}+\delta^{ \pm} \tilde{X}_{\pi}^{L},
\end{aligned}
$$

both for classical and quantum dynamics. Accordingly, we should have to admit that the polarizations mentioned above excluding $\tilde{X}_{\epsilon^{\mp}}^{L}$ :

$$
\begin{aligned}
\mathcal{P}^{+} & =<\tilde{X}_{c^{+}}^{L}, \tilde{X}_{\epsilon^{0}}^{L}, \tilde{X}_{\epsilon^{+}}^{L}> \\
& \text { or } \\
\mathcal{P}^{-} & =<\tilde{X}_{c^{-}}^{L}, \tilde{X}_{\epsilon^{0}}^{L}, \tilde{X}_{\epsilon^{-}}^{L}>,
\end{aligned}
$$

where $c^{ \pm}$are linear combinations of $\kappa, \pi$ diagonalizing $\tilde{X}_{\epsilon^{0}}^{L}$, are now full and symplectic polarizations (for $k \neq 0$ ), so that only $\tilde{X}_{\epsilon^{0}}^{L}$ should be expected to be expressible in terms of basic operators. However, and this is the remembrance of the underlying anomaly, one finds that for some specific values of $a, k$, actually arbitrary $a$ and a certain $k=k(a)$, the "basic" operators $\tilde{X}_{\epsilon^{ \pm}}^{R}$ turn out to be rewritten, accidentally, as functions of the originally basic operators $\tilde{X}_{\kappa, \pi}^{R}$ or $\tilde{X}_{c^{ \pm}}^{R}$. Those specific values of $a, k$ are the quantum values of the anomaly, as opposed to the classical values $a$ arbitrary, $k=0$. We thus feel that the non-existence of a full and symplectic polarization, for some values of the (pseudo-)extension parameters, is more a characteristic of the anomaly phenomenon than the appearance of deformed terms in the Lie algebra.

\subsection{Higher-order Polarizations}

In general, to tackle situations like those mentioned above, it is necessary to generalize the notion of polarization. Let us consider the universal enveloping algebra of left-invariant vector fields, $\mathcal{U} \tilde{\mathcal{G}}^{L}$. We define a higher-order 
polarization $\mathcal{P}^{H O}$ as a maximal subalgebra of $\mathcal{U} \tilde{\mathcal{G}}^{L}$ with no intersection with the abelian subalgebra of powers of $\Xi$. With this definition a higherorder polarization contains the maximal number of conditions compatible with the equivariance condition of the wave functions and with the action of the physical operators (right-invariant vector fields).

We notice that now the vector space of functions annihilated by a higherorder polarization is not, in general, a ring of functions and therefore there is no corresponding foliation; that is, they cannot be characterized by saying that they are constant along submanifolds. If this were the case, it would mean that the higher-order polarization was the enveloping algebra of a firstorder polarization and, accordingly, we could consider the submanifolds associated with this polarization. In this sense the concept of higher-order polarization generalizes and may replace that of first-order polarization.

We arrive at the formulation of our main general proposition, which had been proved only for the particular case of the Virasoro group [24]. We should stress that the introduction of the (left and right) enveloping algebras implies the use of pseudo-differential operators. However, there can be non-trivial operators acting on the Hilbert space of wave functions which are not pseudodifferential, and therefore are not contained in the enveloping algebras. This imply that higher-order polarizations will provide only a Hilbert space on which any differential or pseudo-differential operator commuting with the representation is a multiple of the identity, but possibly containing invariant subspaces which are undistinguishable under the action of all the operators in the group. Such a representation will be called quasi-irreducible.

Proposition: Let $\mathcal{P}^{H O}$ be a higher-order polarization on $\tilde{G}$. On subspaces characterized by

$$
L_{\Xi} \psi=i \psi, \quad A . \psi=0 \quad \forall A \in \mathcal{P}^{H O},
$$

all the right-invariant vector fields $\tilde{X}^{R}$ act quasi-irreducibly. Therefore the present quantization procedure gives rise to a quasi-irreducible representation of the group $\tilde{G}$, provided it is connected and simply connected.

The proof uses the fact that in a canonical chart at the identity, the group law $g^{\prime \prime}=g^{\prime} * g$ proves to be a formal group law 35 and any translation (composition) on the group admits a unique formal power-series expansion, as well as the following

Lemma: If $\hat{O}$ is a pseudo-differential operator acting on the representation space in such a way that $\left[\hat{O}, \tilde{X}^{R}\right]=0$ for any right-invariant vector field, 
then the operator $\hat{O}$ has necessarily the form $\sum_{n=1}^{\infty} a_{i_{1} i_{2} \ldots i_{n}} \tilde{X}_{i_{1}}^{L} \tilde{X}_{i_{2}}^{L} \ldots \tilde{X}_{i_{n}}^{L}, i_{k}=$ $1, \ldots, \operatorname{dim} \tilde{G}$, where the coefficients $a_{i_{1} i_{2} \ldots i_{n}}$ are constants.

The Lemma is a direct consequence of the triviality of the tangent bundle to any Lie group. In fact, any first order operator on the group can be written as $\tilde{X}=a^{i} \tilde{X}_{i}^{L}$, where $\left\{a^{i}\right\}$ are arbitrary functions on the group and $\left\{\tilde{X}_{i}^{L}\right\}$ a basis for the free module of vector fields. Since right- and leftinvariant vector fields commute on any Lie group, the condition $\left[\tilde{X}, \tilde{X}_{i}^{R}\right]=0$ necessarily implies $a^{i}=$ const, $\forall i$. The same condition is obtained for a higher-order operator, which can be written as $\sum_{n=1}^{\infty} a_{i_{1} i_{2} \ldots i_{n}} \tilde{X}_{i_{1}}^{L} \tilde{X}_{i_{2}}^{L} \ldots \tilde{X}_{i_{n}}^{L}$.

Proof of the Proposition: let us assume that the set of solutions of the equations (83) carries a reducible (and not quasi-irreducible) representation of the Lie algebra of $\tilde{G}$ realized by an infinitesimal left action of the group. Therefore, and according to Schur's lemma in it inverse form (see e.g. the classical book by Wigner [36]), a non-trivial (not a multiple of the identity) pseudo-differential operator $\hat{O}$ must exist, at least, which commutes with the representation. The previous Lemma then requires the operator $\hat{O}$ to have the expression given above, and the fact that this operator preserves (it commutes with the representation) the solution space of the polarization $\mathcal{P}^{H O}$ just states its compatibility with the operators in $\mathcal{P}^{H O}$, i.e. it closes an algebra with the elements in the polarization, which, in addition, satisfies the conditions for a higher-order polarization. In fact, if $A$ is any operator in $\mathcal{P}^{H O}$, then

$$
[\hat{O}, A] \psi_{\alpha}=\hat{O} A \psi_{\alpha}-\sum O_{\alpha \beta} A \psi_{\beta}=0,
$$

so that, the right hand side of this equation can only be replaced, at most, by another element of the polarization $A^{\prime}$. In particular, the commutator $[\hat{O}, A]$ will never be proportional to the vertical generator $\Xi$. However, this is precisely the condition for an element in the left enveloping algebra to enter the (higher-order) polarization. Thus, the existence of such a non-trivial operators would imply that $\mathcal{P}^{H O}$ was not maximal.

In the case of infinite-dimensional representations, the validity of Schur's lemma requires the unitarity of the representation, a fact that will restrict, in general, the values of the central charges, as mentioned above.

The definition of higher-order polarization given above is quite general. In all studied examples higher-order polarizations adopt a more definite structure closely related to given first-order (non-full and/or non-symplectic) ones. According to the until now studied cases, higher-order polarizations can be 
given a more operative definition: A higher-order polarization is a maximal subalgebra of $\mathcal{U} \tilde{\mathcal{G}}^{L}$ the "vector field content" of which is a first order polarization. By "vector field content" of a subalgebra $\mathcal{A}$ of $\mathcal{U} \tilde{\mathcal{G}}^{L}$ we mean the following: Let $V(\mathcal{A})$ be the vector space of complex functions on $\tilde{G}$ defined by

$$
V(\mathcal{A})=\left\{f \in \mathcal{F}_{C}(\tilde{G}) / A \cdot f=0, \forall A \in \mathcal{A}\right\} .
$$

Now we generate a ring $R(\mathcal{A})$ by taking any function of elements of $V(\mathcal{A})$. With $R(\mathcal{A})$ we associate the set of left-invariant vector fields defined by

$$
L_{\tilde{X}^{L}} h=0, \quad \forall h \in R(\mathcal{A}) .
$$

This set of left-invariant vector fields is a Lie subalgebra of $\tilde{\mathcal{G}}^{L}$ and defines the vector field content of $\mathcal{A}$, which proves to be a first-order polarization.

A simple example suggesting the generalization of the concept of higherorder polarization corresponds to the non-irreducible representation associated with the non-symplectic polarization (99) of the Schrodinger group (see above). This polarization cannot be further reduced by enlarging (99) to a higher-order polarization $\mathcal{P}^{H O}$. A full reduction requires the inclusion in $\mathcal{P}^{H O}$ of the parity operator commuting with the representation. The generalization of the concept of higher-order polarization so as to include nonpseudo-differential operators, as well as a constructive characterization of those operators deserves a separate study.

In order to throw some light on the structure of higher-order polarizations, let us consider the set $\mathcal{S}$ of all possible higher-order polarizations on $\tilde{G}$. This is a quite large set, so let us try to find some relations on it.

Suppose that $\mathcal{P}^{H O}$ and $\mathcal{P}^{H O \prime}$ are two different higher-order polarizations on $\tilde{G}$, but leading to unitarily equivalent representations of $\tilde{G}$, that is,

$$
\begin{aligned}
\mathcal{P}^{H O} & \Rightarrow U(g) \text { acting on the Hilbert space } \mathcal{H}, \\
\mathcal{P}^{H O \prime} & \Rightarrow U^{\prime}(g) \text { acting on the Hilbert space } \mathcal{H}^{\prime},
\end{aligned}
$$

in such a way that there exists a unitary operator $V: \mathcal{H} \rightarrow \mathcal{H}^{\prime}$, with $U^{\prime}=$ $V U V^{-1}$. Then, it is clear that any element $A^{\prime} \in \mathcal{P}^{H O \prime}$ can be obtained as $A^{\prime}=V A V^{-1}$, for some $A \in \mathcal{P}^{H O}$. Obviously, this introduces an equivalence relation in $\mathcal{S}$, and a partition of it in equivalence classes $\left[\mathcal{P}^{H O}\right]$, where all elements in $\left[\mathcal{P}^{H O}\right]$ are related through unitary transformations.

Let $\mathcal{P}^{1}$ be a fisrt-order polarization (either horizontal or non-horizontal, but always full and symplectic), such that it leads to a (quasi-) irreducible 
representation of $\tilde{G}$ (this means that no anomaly is present). Define $\mathcal{U} \mathcal{P}^{1}$ as the enveloping algebra of $\mathcal{P}^{1}$ by the whole $\mathcal{U} \tilde{\mathcal{G}}^{L}$, that is,

$$
\mathcal{U} \mathcal{P}^{1} \equiv\left\{A=\sum_{k=1}^{m} A_{k} \tilde{X}_{k}^{L}, \text { such that } A_{k} \in \mathcal{U} \tilde{\mathcal{G}}^{L}\right\}
$$

where $\left\{\tilde{X}_{k}^{L}\right\}_{k=1}^{m}$ is a basis for $\mathcal{P}^{1}$, and $m=\operatorname{dim} \mathcal{P}^{1}$. Then it is easy to check that $\mathcal{U} \mathcal{P}^{1}$ is a higher-order polarization. In fact, it does not contain the vertical generator $\Xi$ (nor its powers) because $\mathcal{P}^{1}$ does not contain it either, and $\mathcal{U} \mathcal{P}^{1}$ it is maximal, otherwise the representation obtained with $\mathcal{P}^{1}$ would not be (quasi)-irreducible.

Consider the class $\left[\mathcal{U} \mathcal{P}^{1}\right]$ of all higher-order polarizations in $\mathcal{S}$ unitarily equivalent to $\mathcal{U} \mathcal{P}^{1}$. For certain groups, as for instance, (finite) semisimple and solvable groups [3], it is always possible to find an "admissible" subalgebra associated with any quantization 1-form $\Theta$ or, in other words, it is always possible to find a full and symplectic first-order polarization. This means that for these groups $\mathcal{S}=\cup_{\alpha \in I}\left[\mathcal{U P}^{1}\right]_{\alpha}$, where the set $I$ parameterizes the (quasi-)irreducible representations of $\tilde{G}$ (which are associated to coadjoint orbits).

Note that, from the very construction of $\mathcal{U} \mathcal{P}^{1}$, it admits a basis (of vector fields in this case), finite for finite-dimensional groups, and this structure is translated to all $\mathcal{P}^{H O} \in\left[\mathcal{U} \mathcal{P}^{1}\right]$, the basis being of the form $\left\{V \tilde{X}_{k}^{L} V^{-1}\right\}_{k=1}^{m}$. For this kind of higher-order polarizations, this allows us to define the $d i$ mension of a higher-order polarization as the dimension of the first-order polarization to which it is equivalent. Although we do not give a proof, it is reasonable that all higher-order polarizations, even if they are not equivalent to a first-order one, admit a finite basis (for finite-dimensional groups), and this is what happens, for instance, with the anomalous Schrödinger and Virasoro groups (this last example admits an infinite basis, since the group is infinite dimensional, but it is countable).

Finally, let us comment on the structure of the wave functions that are solutions of a higher-order polarization. For the case of a first-order polarization, the solutions of $\tilde{X}^{L} \Psi=0, \forall \tilde{X}^{L} \in \mathcal{P}^{1}$ have always the form

$$
\Psi=\zeta e^{i \chi(g)} \Phi
$$

where $\chi(g)$ is a real function on $G$. The functions $\Phi$ are defined on a Lagrangian submanifold $S$ of $\tilde{G}$ (with respect to $d \Theta$ ). We can think of the 
unitary operator $e^{i \chi(g)}$ as relating the representation in terms of the rightinvariant vectorfields $\tilde{X}^{R}$ acting on the subspace of $L^{2}(\tilde{G})$ of polarized functions, to the representation in terms of the (first-order) differential operators $e^{-i \chi(g)} \tilde{X}^{R} e^{i \chi(g)}$ acting on the Hilbert space $L^{2}(S)$. Note that the unitary operator $e^{i \chi(g)}$ is diagonal, and this is related to the fact that $S$ is a Lagrangian submanifold of $\tilde{G}$.

However, for higher-order polarizations, the wave functions, solutions of $A \Psi=0, \forall A \in \mathcal{P}^{H O}$, do not define a foliation in $\tilde{G}$. But they can be written, at least formally, as:

$$
\Psi=\zeta e^{i \hat{O}} \Phi
$$

where $\hat{O}$ is a pseudo-differential operator on $\tilde{G}$. The reason is that, in general, the polarization equations have the form, or can be taken (formally) to the form:

$$
i \frac{\partial}{\partial g^{s}} \Psi=\hat{O}_{s} \Psi, s=1, \ldots, k,
$$

where $k$ is the dimension of $\mathcal{P}^{H O}$. The function $\Phi$ belongs to $L^{2}(S)$, although $S$ is not a Lagrangian submanifold of $\tilde{G}$.

As in the case of first-order polarizations, we can think of the unitary operator $e^{i \hat{O}}$ as stating the equivalence of the representation defined by the right-invariant vector fields $\tilde{X}^{R}$, acting on the subspace of $L^{2}(\tilde{G})$ defined by the polarized wave functions, and the representation defined by the (higherorder) differential operators $e^{-i \hat{O}} \tilde{X}^{R} e^{i \hat{O}}$, acting on $L^{2}(S)$.

To see how a higher-order polarization operates in practice, we shall consider the examples of the anomalous Schrödinger and Virasoro groups.

\subsection{The Schrödinger group}

To illustrate the Lie algebra structure of an anomalous group, let us consider the example of the Schrödinger group in one dimension. This group, or rather, the complete Weyl-Symplectic group, was considered in Ref. [3 as an example of a group not possessing an "admissible" subalgebra. It is the semidirect action of the $S L(2, R)$ group on the H-W group (when considered in $n$ dimensions, rotations should also be included) including as subgroups the symmetry group of the free particle, the Galilei group, as well as the symmetry group of the ordinary harmonic oscillator and the "repulsive" harmonic oscillator (with imaginary frequency), usually known as Newton 
groups [37. From the mathematical point of view, it can be obtained from the Galilei group (or from either of the Newton groups) by replacing the time subgroup with the three-parameter group $S L(2, R)$. In fact, those kinematical subgroups are associated with different choices of a Hamiltonian inside $S L(2, R)$.

In order to perform a global-coordinate treatment of the problem, we shall start by considering matrices $S \in G L(2, R)$ instead of $S L(2, R)$, and the condition for these matrices to belong to $S L(2, R)$ will appear naturally. A group law for the Schrödinger group can be written as:

$$
\begin{aligned}
\vec{x}^{\prime \prime} & =|S|^{1 / 2} S^{-1} \vec{x}^{\prime}+\vec{x} \\
S^{\prime \prime} & =S^{\prime} S \\
\zeta^{\prime \prime} & =\zeta^{\prime} \zeta \exp \frac{i m \omega}{2 \hbar}\left[\frac{-A x_{2}^{\prime} x_{1}-B x_{2}^{\prime} x_{2}+C x_{1}^{\prime} x_{1}+D x_{1}^{\prime} x_{2}}{|S|^{1 / 2}}\right],
\end{aligned}
$$

where $\vec{x}=\left(x_{1}, x_{2}\right) \in R^{2}, S=\left(\begin{array}{cc}A & B \\ C & D\end{array}\right) \in G L(2, R),|S| \equiv A D-B C$ and $\frac{m \omega}{\hbar}$ is a constant parametrizing the central extensions of the $\mathrm{H}-\mathrm{W}$ group (we write it in this form for later convenience). The factor $|S|^{1 / 2}$ in the semidirect action of $G L(2, R)$ is needed in order to have a proper central extension. For the moment, we shall assign no dimensions to $x_{1}$ and $x_{2}$, but if we want to identify them with $q$ and $v$ of the $\mathrm{H}-\mathrm{W}$ group in section 1.1, and keep $S L(2, R)$ adimensional, then it should be $x_{1}=q$ and $x_{2}=v / \omega$, where $\omega$ is a constant having the dimensions of a frequency $\left(T^{-1}\right)$.

From the group law, the left-invariant vector fields associated with the coordinates $x_{1}, x_{2}, A, B, C, D, \zeta$,

$$
\begin{aligned}
\tilde{X}_{x_{1}}^{L} & =\frac{\partial}{\partial x_{1}}-\frac{m \omega}{2 \hbar} x_{2} \Xi \\
\tilde{X}_{x_{2}}^{L} & =\frac{\partial}{\partial x_{2}}+\frac{m \omega}{2 \hbar} x_{1} \Xi \\
\tilde{X}_{A}^{L} & =A \frac{\partial}{\partial A}+C \frac{\partial}{\partial C}-\frac{1}{2} x_{1} \frac{\partial}{\partial x_{1}}+\frac{1}{2} x_{2} \frac{\partial}{\partial x_{2}} \\
\tilde{X}_{B}^{L} & =A \frac{\partial}{\partial B}+C \frac{\partial}{\partial D}-x_{2} \frac{\partial}{\partial x_{1}} \\
\tilde{X}_{C}^{L} & =B \frac{\partial}{\partial A}+D \frac{\partial}{\partial C}-x_{1} \frac{\partial}{\partial x_{2}}
\end{aligned}
$$




$$
\begin{aligned}
\tilde{X}_{D}^{L} & =B \frac{\partial}{\partial B}+D \frac{\partial}{\partial D}+\frac{1}{2} x_{1} \frac{\partial}{\partial x_{1}}-\frac{1}{2} x_{2} \frac{\partial}{\partial x_{2}} \\
\tilde{X}_{\zeta}^{L} & =i \zeta \frac{\partial}{\partial \zeta} \equiv \Xi,
\end{aligned}
$$

as well as the right-invariants ones,

$$
\begin{aligned}
\tilde{X}_{x_{1}}^{R} & =|S|^{-1 / 2}\left[D \frac{\partial}{\partial x_{1}}-C \frac{\partial}{\partial x_{2}}+\frac{m \omega}{2 \hbar}\left(D x_{2}+C x_{1}\right) \Xi\right] \\
\tilde{X}_{x_{2}}^{R} & =|S|^{-1 / 2}\left[A \frac{\partial}{\partial x_{2}}-B \frac{\partial}{\partial x_{1}}-\frac{m \omega}{2 \hbar}\left(B x_{2}+A x_{1}\right) \Xi\right] \\
\tilde{X}_{A}^{R} & =A \frac{\partial}{\partial A}+B \frac{\partial}{\partial B} \\
\tilde{X}_{B}^{R} & =D \frac{\partial}{\partial B}+C \frac{\partial}{\partial A} \\
\tilde{X}_{C}^{R} & =A \frac{\partial}{\partial C}+B \frac{\partial}{\partial D} \\
\tilde{X}_{D}^{R} & =C \frac{\partial}{\partial C}+D \frac{\partial}{\partial D} \\
\tilde{X}_{\zeta}^{R} & =\Xi
\end{aligned}
$$

can be obtained. The commutation relations for the (left) Lie algebra are:

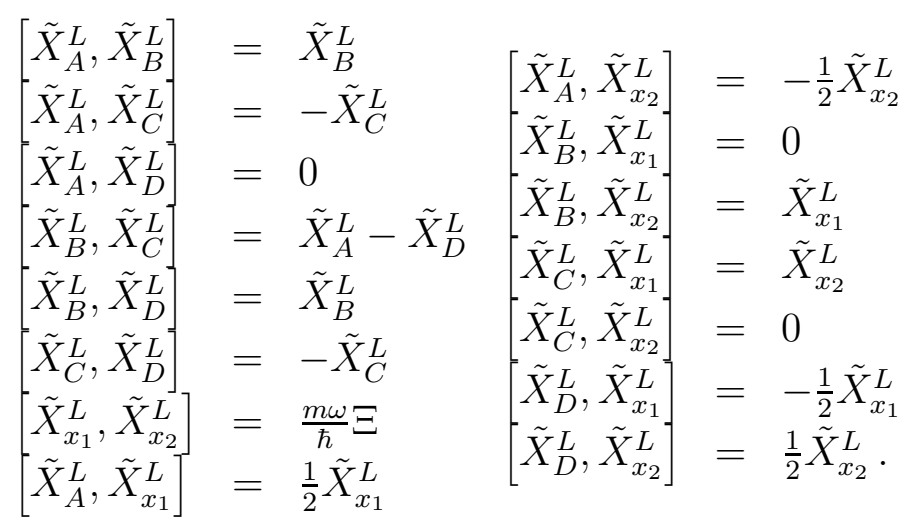

From these commutation relations we see that two linear combinations of vector fields can be introduced, $\tilde{X}_{A}^{L}-\tilde{X}_{D}^{L}$ and $\tilde{X}_{A}^{L}+\tilde{X}_{D}^{L}$ (the same for the right-invariant vector fields), in such a way that $\tilde{X}_{A}^{L}+\tilde{X}_{D}^{L}$ is a central generator, which is also horizontal, and, therefore, it is a gauge generator 
(see [32], and also subsection 4.2, where something analogous happens for the case of the $S U(2)$ group). In fact, it coincides with its right version, as is always the case for a central generator.

The quantization 1-form $\Theta$ is:

$$
\begin{aligned}
\Theta= & \frac{m \omega}{2 \hbar}\left(x_{2} d x_{1}-x_{1} d x_{2}+\frac{1}{|S|}\left[C x_{1}^{2} d A+D x_{2}^{2} d B\right.\right. \\
& \left.\left.-A x_{1}^{2} d C-B x_{2}^{2} d D+x_{1} x_{2} d|S|\right]\right)+\frac{d \zeta}{i \zeta}
\end{aligned}
$$

and the characteristic subalgebra has the form:

$$
\mathcal{G}_{\Theta}=<\tilde{X}_{A}^{L}+\tilde{X}_{D}^{L}, \tilde{X}_{A}^{L}-\tilde{X}_{D}^{L}, \tilde{X}_{B}^{L}, \tilde{X}_{C}^{L}>
$$

The Noether invariants, as introduced in Sec. 3, turn out to be:

$$
\begin{array}{ll}
F_{A}=\frac{m \omega}{2 \hbar} x_{1} x_{2} & F_{D}=-\frac{m \omega}{2 \hbar} x_{1} x_{2} \\
F_{B}=\frac{m \omega}{2 \hbar} x_{2}^{2} & F_{x_{1}}=\frac{m \omega}{\hbar} x_{2} \\
F_{C}=-\frac{m \omega}{2 \hbar} x_{1}^{2} & F_{x_{2}}=-\frac{m \omega}{\hbar} x_{1},
\end{array}
$$

revealing that the Noether invariant associated with the right-invariant vector fields whose left counterpart lie in the characteristic subalgebra can be expressed in terms of the basic Noether invariants $F_{x_{1}}$ and $F_{x_{2}}$ :

$$
\begin{array}{ll}
F_{A}+F_{D}=0 & F_{B}=\frac{\hbar}{2 m \omega} F_{x_{2}}^{2} \\
F_{A}-F_{D}=-\frac{\hbar}{m \omega} F_{x_{1}} F_{x_{2}} & F_{C}=-\frac{\hbar}{2 m \omega} F_{x_{1}}^{2},
\end{array}
$$

Note also that the Noether invariant associated with the generator $\tilde{X}_{A}^{R}+\tilde{X}_{D}^{R}$ is zero, as corresponds to a gauge generator [32]. These relations between Noether invariants lead to a phase space of the classical theory of dimension two (i.e. only one degree of freedom). We have obtained this result because the $S L(2, R)$ subgroup is represented trivially, that is, the twococycle we have chosen for the Schrödinger group lies in the trivial pseudocohomology class, associated with the zero-dimensional coadjoint orbit of $S L(2, R)$. Therefore, only the 2-dimensional coadjoint orbit of the $\mathrm{H}-\mathrm{W}$ group is taken into account. See [38, 39] for the study of the Schrödinger group considering all coadjoint orbits of $S L(2, R)$.

As we have seen, the Lie-algebra two-cocycle $\Sigma$ contains the entire $S L(2, R)$ subalgebra (together with the gauge generator) in its kernel, which, according to the general scheme, should enter any full and symplectic polarization. 
Unfortunately such a polarization does not exist, and this can be traced back to the fact that the partial complex structure $J=\theta^{L x_{1}} \otimes \tilde{X}_{x_{2}}^{L}-\theta^{L x_{2}} \otimes \tilde{X}_{x_{1}}^{L}$ is not preserved by its kernel (the $S L(2, R)$ subalgebra), implying that we cannot relate it to a complex structure on the classical phase-space. We can only find a non-symplectic, full polarization,

$$
\mathcal{P}_{C}=<\tilde{X}_{A}^{L}+\tilde{X}_{D}^{L}, \tilde{X}_{A}^{L}-\tilde{X}_{D}^{L}, \tilde{X}_{B}^{L}, \tilde{X}_{C}^{L}>,
$$

and a series of non-full, but symplectic polarizations. The latters are obtained by adding, to the polarizations given in section 4.1 for the $\mathrm{H}-\mathrm{W}$ group, the gauge generator $\tilde{X}_{A}^{L}+\tilde{X}_{D}^{L}$ and two (properly selected) generators out of the $S L(2, R)$ subgroup.

Quantizing with these non-full polarizations results in a breakdown of the naively expected relations between the operators $\tilde{X}_{A}^{R}-\tilde{X}_{D}^{R}, \tilde{X}_{B}^{R}, \tilde{X}_{C}^{R}$ and the basic ones $\tilde{X}_{x_{1}}^{R}, \tilde{X}_{x_{2}}^{R}$, as suggested by the relations between Noether invariants (98).

Furthermore, quantizing with the non-symplectic polarization (99) leads to an unconventional representation in which the wave functions depend on both $x_{1}$ and $x_{2}$ variables, and contain two irreducible components (see [1]). Also, neither of the operators $\tilde{X}_{A}^{R}-\tilde{X}_{D}^{R}, \tilde{X}_{B}^{R}$, nor $\tilde{X}_{C}^{R}$, can be expressed in terms of the basic ones.

In all these cases, the operators of $S L(2, R)$ behave as if they had symplectic content; that is, as if they were associated with a new degree of freedom (see [38] and [39] for different discussions about the meaning of this degree of freedom). Thus, there is a breakdown of the correspondence between (classical) coadjoint orbits and (quantum) Hilbert spaces.

Nevertheless, it is still possible to obtain a quantum theory associated with the classical theory given by the Noether invariants (98). And this is given by means of a higher-order polarization of the form:

$$
\begin{aligned}
\mathcal{P}^{H O}= & <\tilde{X}_{A}^{L}+\tilde{X}_{D}^{L}, \tilde{X}_{A}^{L}-\tilde{X}_{D}^{L}-\frac{i \hbar}{2 m \omega}\left(\tilde{X}_{x_{1}}^{L} \tilde{X}_{x_{2}}^{L}+\tilde{X}_{x_{2}}^{L} \tilde{X}_{x_{1}}^{L}\right), \\
& \tilde{X}_{B}^{L}+\frac{i \hbar}{2 m \omega}\left(\tilde{X}_{x_{1}}^{L}\right)^{2}, \tilde{X}_{C}^{L}-\frac{i \hbar}{2 m \omega}\left(\tilde{X}_{x_{2}}^{L}\right)^{2}>\cup \mathcal{P}^{1},
\end{aligned}
$$

where $\mathcal{P}^{1}$ is any of the (first-order) polarizations for the $\mathrm{H}-\mathrm{W}$ group given in section 4.1 (taking there $\mathrm{k}=2$, so that we can forget about the generators $\left.\tilde{X}_{\vec{a}}^{L}\right)$. Note that the higher-order elements of the polarizations correspond 
(apart from normal order) to making the substitution $F_{g}^{i} \rightarrow-i \tilde{X}_{g^{i}}^{L}$ in the relations among Noether invariants (98). It should be stressed that the normal order is automatically fixed, precisely by the requirement for $\mathcal{P}^{H O}$ of being a higher-order polarization. In more general situations, there will be different possibilities in the normal order, in correspondence with different choices of higher-order polarizations.

For each choice of $\mathcal{P}^{1}$, it is more convenient to express the higher-order terms in a different basis, to obtain the representation in the adequate parameters for the description of the free particle, the harmonic oscillator or the repulsive oscillator. Thus, the different higher-order polarizations could be:

$$
\begin{aligned}
\mathcal{P}_{v}^{H O}= & <\tilde{X}_{A}^{L}+\tilde{X}_{D}^{L}, \tilde{X}_{A}^{L}-\tilde{X}_{D}^{L}-\frac{i \hbar}{2 m \omega}\left(\tilde{X}_{x_{1}}^{L} \tilde{X}_{x_{2}}^{L}+\tilde{X}_{x_{2}}^{L} \tilde{X}_{x_{1}}^{L}\right), \\
& \tilde{X}_{B}^{L}+\frac{i \hbar}{2 m \omega}\left(\tilde{X}_{x_{1}}^{L}\right)^{2}, \tilde{X}_{C}^{L}-\frac{i \hbar}{2 m \omega}\left(\tilde{X}_{x_{2}}^{L}\right)^{2}, \tilde{X}_{x_{1}}^{L}>, \\
\mathcal{P}_{c}^{H O}= & <\tilde{X}_{A}^{L}+\tilde{X}_{D}^{L},\left(\tilde{X}_{A}^{L}-\tilde{X}_{D}^{L}\right)+i\left(\tilde{X}_{B}^{L}-\tilde{X}_{C}^{L}\right)-\frac{\hbar}{m \omega}\left(\tilde{X}_{c}^{L}\right)^{2}, \\
& \left(\tilde{X}_{A}^{L}-\tilde{X}_{D}^{L}\right)-i\left(\tilde{X}_{B}^{L}-\tilde{X}_{C}^{L}\right)+\frac{\hbar}{m \omega}\left(\tilde{X}_{c^{*}}^{L}\right)^{2}, \\
& \tilde{X}_{B}^{L}-\tilde{X}_{C}^{L}+\frac{i \hbar}{2 m \omega}\left(\tilde{X}_{c}^{L} \tilde{X}_{c^{*}}^{L}+\tilde{X}_{c^{*}}^{L} \tilde{X}_{c}^{L}\right), \tilde{X}_{c^{*}}^{L}>, \\
\mathcal{P}_{\rho_{-}}^{H O}= & <\tilde{X}_{A}^{L}+\tilde{X}_{D}^{L},\left(\tilde{X}_{A}^{L}-\tilde{X}_{D}^{L}\right)+\left(\tilde{X}_{B}^{L}-\tilde{X}_{C}^{L}\right)+\frac{i \hbar}{m \omega}\left(\tilde{X}_{\rho_{-}}^{L}\right)^{2}, \\
& \left(\tilde{X}_{A}^{L}-\tilde{X}_{D}^{L}\right)-\left(\tilde{X}_{B}^{L}-\tilde{X}_{C}^{L}\right)-\frac{i \hbar}{m \omega}\left(\tilde{X}_{\rho_{+}}^{L}\right)^{2}, \\
& \tilde{X}_{B}^{L}+\tilde{X}_{C}^{L}+\frac{i \hbar}{2 m \omega}\left(\tilde{X}_{\rho_{+}}^{L} \tilde{X}_{\rho_{-}}^{L}+\tilde{X}_{\rho_{-}}^{L} \tilde{X}_{\rho_{+}}^{L}\right), \tilde{X}_{\rho_{+}}^{L}>.
\end{aligned}
$$

It should be stressed that all these higher-order polarizations are equivalent, in the sense that they lead to (unitarily) equivalent representations of the Schrödinger group. However, one can select certain uniparametric subgroups of $S L(2, R)$ to assign a dynamics to the H-W group, and obtain in this way three differents subgroups of the Schrödinger group: the Galilei group of the free non-relativistic particle, the harmonic oscillator group, and the repulsive harmonic oscillator group. The three different higher-order polarizations $\mathcal{P}_{v}^{H O}, \mathcal{P}_{c}^{H O}$ and $\mathcal{P}_{\rho_{-}}^{H O}$ are adequate for the description of each 
one of these systems because they incorporate, explicitely, the corresponding Schrödinger equation, as a polarization equation, which turns out to lead to a first-order differential equation.

Let us obtain the representation associated with $\mathcal{P}_{v}^{H O}$. The polarization equations are:

$$
\begin{aligned}
\left(\tilde{X}_{A}^{L}+\tilde{X}_{D}^{L}\right) \Psi & =0 \\
\tilde{X}_{B}^{L} \Psi & =0 \\
\left(\tilde{X}_{A}^{L}-\tilde{X}_{D}^{L}\right) \Psi & =-\frac{1}{2} \Psi \\
\tilde{X}_{x_{1}}^{L} \Psi & =0 \\
\tilde{X}_{C}^{L} \Psi & =\frac{i \hbar}{2 m \omega}\left(\tilde{X}_{x_{2}}^{L}\right)^{2} \Psi
\end{aligned}
$$

The first of these equations has as solutions those complex wave functions on the group $G L(2, R)$, which are defined on $S L(2, R)$, as expected. Therefore, the solutions of this equation have the form (once the usual $U(1)$ equivariant condition is imposed):

$$
\Psi=\zeta \Phi\left(a, b, c, d, x_{1}, x_{2}\right)
$$

where $a \equiv \frac{A}{\sqrt{A D-B C}}, b \equiv \frac{B}{\sqrt{A D-B C}}, c \equiv \frac{C}{\sqrt{A D-B C}}$ and $d \equiv \frac{D}{\sqrt{A D-B C}}$, with $a d-b c=1$.

To proceed further in solving the polarization equations, it is convenient to introduce local charts on $S L(2, R)$. We choose them ads the ones defined by $a \neq 0$ and $c \neq 0$, respectively 1 . The first chart contains the identity element $I_{2}$ of $S L(2, R)$, and the second contains $J \equiv\left(\begin{array}{cc}0 & 1 \\ -1 & 0\end{array}\right)$.

The solutions to the the polarization equations are given by:

- For $a \neq 0$ :

$$
\Psi=\zeta a^{-1 / 2} e^{\frac{i m \omega}{2 \hbar} x y} \chi(\tau, y),
$$

\footnotetext{
${ }^{1}$ Certainly they really correspond to four contractible charts: $a>0, a<0$ and $c<$ $0, c>0$, but the transition functions between each pair of these charts are trivial, so that we shall consider them as only one chart.
} 
where $x \equiv a\left(x_{1}+\frac{b}{a} x_{2}\right), y \equiv x_{2} / a$ and $\tau \equiv \frac{c}{a}$, with $\chi$ satisfying the Schrödinger-like equation

$$
\frac{\partial \chi}{\partial \tau}=\frac{i \hbar}{2 m \omega} \frac{\partial^{2} \chi}{\partial y^{2}}
$$

- For $c \neq 0$ :

$$
\widetilde{\Psi}=\zeta c^{-1 / 2} e^{-\frac{i m \omega}{2 \hbar} \tilde{x} \tilde{y}} \tilde{\chi}(\tilde{\tau}, \tilde{y})
$$

where $\tilde{x} \equiv x_{2} / c, \tilde{y} \equiv a\left(x_{1}-\tilde{\tau} x_{2}\right)$ and $\tilde{\tau} \equiv \frac{a}{c}$, with $\tilde{\chi}$ satisfying the Schrödinger-like equation

$$
\frac{\partial \widetilde{\chi}}{\partial \tilde{\tau}}=-\frac{i \hbar}{2 m \omega} \frac{\partial^{2} \tilde{\chi}}{\partial \tilde{y}^{2}}
$$

The element $J$ represents a rotation of $\frac{\pi}{2}$ in the plane $\left(x_{1}, x_{2}\right)$, and takes the wave function from one local chart to the other ${ }^{2}$. Obviously, $J^{4}=I_{2}$, but acting with $J$ on the wave functions we obtain:

$$
\Psi(J * g)=(-1)^{1 / 4} \widetilde{\Psi}(g),
$$

from which the result $\Psi\left(J^{4} * g\right)=-\Psi(g)$ follows; that is, the representation obtained for the subgroup $S L(2, R)$ is two-valued. This representation is the well-known metaplectic or spinor representation. The metaplectic representation is for $S L(2, R)$ what the $\frac{1}{2}$-spin representation is for $S O(3)$ (see 40 and references therein, and also [3]).

If now one computes the action of the right-invariant vector fields on these wave functions (now we shall forget about the second chart, since we simply have to "Fourier"-transform with $J$ to obtain the results in the other chart), we obtain:

$$
\begin{aligned}
& \tilde{X}_{x_{1}}^{R} \Psi=\zeta a^{-1 / 2} e^{\frac{i m \omega}{2 \hbar} x y}\left[i \frac{m \omega}{\hbar} y-\tau \frac{\partial}{\partial y}\right] \chi(\tau, y) \\
& \tilde{X}_{x_{2}}^{R} \Psi=\zeta a^{-1 / 2} e^{\frac{i m \omega}{2 \hbar} x y}\left[\frac{\partial}{\partial y}\right] \chi(\tau, y)
\end{aligned}
$$

\footnotetext{
${ }^{2}$ In fact, up to a factor, $J$ represents the Fourier transform passing from the $x_{1}$ representation to the $x_{2}$ representation.
} 


$$
\begin{aligned}
\left(\tilde{X}_{A}^{R}-\tilde{X}_{D}^{R}\right) \Psi & =\zeta a^{-1 / 2} e^{\frac{i m \omega}{2 \hbar} x y}\left[-y \frac{\partial}{\partial y}-\frac{1}{2}-\tau \frac{\partial}{\partial \tau}\right] \chi(\tau, y) \\
\tilde{X}_{B}^{R} \Psi & =\zeta a^{-1 / 2} e^{\frac{i m \omega}{2 \hbar} x y}\left[i \frac{m \omega}{2 \hbar} y^{2}-\tau^{2} \frac{\partial}{\partial \tau}-\tau\left(y \frac{\partial}{\partial y}+\frac{1}{2}\right)\right] \chi(\tau, y) \\
\tilde{X}_{C}^{R} \Psi & =\zeta a^{-1 / 2} e^{\frac{i m \omega}{2 \hbar} x y}\left[\frac{\partial}{\partial \tau}\right] \chi(\tau, y) .
\end{aligned}
$$

It is easy to check that the full reduction has been achieved, since we can reproduce the relation between Noether invariants:

$$
\begin{aligned}
\tilde{X}_{B}^{R} \Psi & =-i \frac{\hbar}{2 m \omega}\left(\tilde{X}_{x_{1}}^{R}\right)^{2} \Psi \\
\tilde{X}_{C}^{R} \Psi & =i \frac{\hbar}{2 m \omega}\left(\tilde{X}_{x_{2}}^{R}\right)^{2} \Psi \\
\left(\tilde{X}_{A}^{R}-\tilde{X}_{D}^{R}\right) \Psi & =i \frac{\hbar}{2 m \omega}\left(\tilde{X}_{x_{1}}^{R} \tilde{X}_{x_{2}}^{R}+\tilde{X}_{x_{2}}^{R} \tilde{X}_{x_{1}}^{R}\right) \Psi .
\end{aligned}
$$

We can give a more compact form to the expressions of the right-invariant vector fields, by solving (formally) the Schrödinger-like equation in (105), so that we can write $\chi(\tau, y)=e^{i \frac{\hbar}{2 m \omega} \tau \frac{d^{2}}{d y^{2}}} \phi(y)$, where now $\phi(y)$ is an arbitrary funtion of $y$, representing the unique degree of freedom of the system. As indicated in Sec. 5.1, we can obtain the expressions for the action of the group $\tilde{G}$ in $L^{2}(S)$, where $S$ is the manifold defined by the coordinate $y$ (in this case $S=R$ ), performing the unitary transformation mentioned there; that is, $X^{H O} \equiv e^{-i \hat{O}} \tilde{X}^{R} e^{i \hat{O}}$, where $e^{i \hat{O}}$ is the unitary operator

$$
e^{i \hat{O}}=a^{-1 / 2} e^{\frac{i m \omega}{2 \hbar} x y} e^{i \frac{\hbar}{2 m \omega} \tau \frac{d^{2}}{d y^{2}}} .
$$

The expresion of the pseudo-differential operators $X^{H O}$ acting on $L^{2}(S)$ can be obtained (with a bit of care, since in general one has to apply the Campbell-Haussdorf formula and the relation $e^{-A} B e^{A}=B+[B, A]+$ $\left.\frac{1}{2 !}[[B, A], A]+\frac{1}{3 !}[[[B, A], A], A]+\cdots\right)$, proving to be:

$$
\begin{aligned}
X_{x_{1}}^{H O} & =i \frac{m \omega}{\hbar} y \\
X_{x_{2}}^{H O} & =\frac{d}{d y}
\end{aligned}
$$




$$
\begin{aligned}
X_{A}^{H O}-X_{D}^{H O} & =-y \frac{d}{d y}-\frac{1}{2} \\
X_{B}^{H O} & =i \frac{m \omega}{2 \hbar} y^{2} \\
X_{C}^{H O} & =i \frac{\hbar}{2 m \omega} \frac{d^{2}}{d y^{2}} .
\end{aligned}
$$

Note that the operator $X_{A}^{H O}-X_{D}^{H O}$ (which is nothing other than the dilation operator) appears with the correct ordering, i.e. it is symmetrized. This fact is traced back to the form of the higher-order polarization $\mathcal{P}_{v}^{H O}$, which imposes the correct normal form to all the operators, and that is uniquely determined by the very definition of higher-order polarization. That is, with the formalism of higher-order polarizations there is no need of imposing symmetrization "by hand" to the final operators.

As commented before, this polarization is adequate for the description of the free non-relativistic particle. We only have to restrict ourselves to the subgroup of matrices $S$ of the form:

$$
S=\left(\begin{array}{rr}
1 & \omega t \\
0 & 1
\end{array}\right)
$$

for which only the generator $\tilde{X}_{B}^{R}$, with $B=\omega t$, of the $S L(2, R)$ is relevant. The parameter $\omega$, introduced in order to have adimensional matrices, will disappear in the final expressions, as expected, since in the Galiei group the only extra parameter is the mass $m$ (and $\hbar$ ). The wave functions, restricted to the Galilei subgroup, are $\Psi=\zeta e^{i \frac{m}{\hbar}(q+t v) v} \phi(v)$, where $q \equiv x_{1}$ and $v \equiv \omega x_{2}$. The action of the right-invariant operators, once they are reduced to the space $L^{2}(S)$ (which in this case, and for the operators of the Galilei subgroup, is expressed in terms of first-order differential operators), is:

$$
\begin{aligned}
\hat{p} \equiv-i \hbar X_{x_{1}}^{H O} & =p \\
\hat{q} \equiv-i \frac{\hbar}{\omega} X_{x_{2}}^{H O} & =-i \hbar \frac{d}{d p} \\
\hat{E} \equiv i \hbar \omega X_{B}^{H O} & =\frac{p^{2}}{2 m},
\end{aligned}
$$

where $p \equiv m v$. The Schrödinger equation is given by the polarization equation $\tilde{X}_{B}^{L} \Psi=0$, and it simply tells us that $i \hbar \frac{\partial}{\partial t} \Psi=\frac{p^{2}}{2 m} \Psi$, as usual. 
The same precedure can be carried out to obtain the solutions to the polarizations $\mathcal{P}_{c}^{H O}$ and $\mathcal{P}_{\vec{\rho}_{-}}^{H O}$, and to obtain the usual expressions for the quantum operators, once we restrict ourselves to the ordinary and repulsive harmonic oscillators, respectively. We shall not pursue on it, and send the reader to Ref. 31] for a detailed study on them, although there the quantization of the complete Schrödinger group was not carried out - only the different subgroups were studied separatedly- since higher-order polarizations were not available yet. This is the reason why in 31 the corrective term $\frac{1}{2}$ was introduced "by hand" in the expressions of the operators like $\tilde{X}_{A}^{R}-\tilde{X}_{D}^{R}$, or in the energy operators of the standard and repulsive oscillators. With the use of higher-order polarizations this is no longer needed, since all operators appear automatically normally ordered. This is an advantage of the technique of higher-order polarizations, which is more natural (we believe) than the "metaplectic correction" (see [11, 40]).

If one wishes to obtain the representation in configuration space for all of these systems, with higher-order differential Schrödinger equations, one simply has to choose $\mathcal{P}_{q}$ for $\mathcal{P}^{1}$, as well as an adequate basis for the higher-order terms in such a way that the corresponding Schrödinger equation appears, explicitely, as one of the polarization equations.

For instance, the higher-order polarization,

$$
\begin{aligned}
\mathcal{P}_{\text {Galilei }}^{H O}= & <\tilde{X}_{A}^{L}+\tilde{X}_{D}^{L}, \tilde{X}_{A}^{L}-\tilde{X}_{D}^{L}-\frac{i \hbar}{2 m \omega}\left(\tilde{X}_{x_{1}}^{L} \tilde{X}_{x_{2}}^{L}+\tilde{X}_{x_{2}}^{L} \tilde{X}_{x_{1}}^{L}\right), \\
& \tilde{X}_{B}^{L}+\frac{i \hbar}{2 m \omega}\left(\tilde{X}_{x_{1}}^{L}\right)^{2}, \tilde{X}_{C}^{L}-\frac{i \hbar}{2 m \omega}\left(\tilde{X}_{x_{2}}^{L}\right)^{2}, \tilde{X}_{x_{2}}^{L}>,
\end{aligned}
$$

would be appropriate for the description of the free non-relativistic particle in configuration space, since it incorporates the second-order Schrödinger equation $\left[\tilde{X}_{B}^{L}+\frac{i \hbar}{2 m \omega}\left(\tilde{X}_{x_{1}}^{L}\right)^{2}\right] \Psi=0$.

In the same way, the higher-order polarization,

$$
\begin{aligned}
\mathcal{P}_{\text {Newton }}^{H O}= & <\tilde{X}_{A}^{L}+\tilde{X}_{D}^{L}, \tilde{X}_{A}^{L}-\tilde{X}_{D}^{L}-\frac{i \hbar}{2 m \omega}\left[\tilde{X}_{x_{1}}^{L} \tilde{X}_{x_{2}}^{L}+\tilde{X}_{x_{2}}^{L} \tilde{X}_{x_{1}}^{L}\right] \\
& \tilde{X}_{B}^{L}-\tilde{X}_{C}^{L}+\frac{i \hbar}{2 m \omega}\left[\left(\tilde{X}_{x_{1}}^{L}\right)^{2}+\left(\tilde{X}_{x_{2}}^{L}\right)^{2}\right] \\
& \tilde{X}_{B}^{L}+\tilde{X}_{C}^{L}+\frac{i \hbar}{2 m \omega}\left[\left(\tilde{X}_{x_{1}}^{L}\right)^{2}-\left(\tilde{X}_{x_{2}}^{L}\right)^{2}\right], \tilde{X}_{x_{2}}^{L}>
\end{aligned}
$$


is adequate for the descrition of the Newton groups, i.e the attractive and the repulsive harmonic oscillators, since it incorporates both Schrödinger equations, $\left\{\tilde{X}_{B}^{L}-\tilde{X}_{C}^{L}+\frac{i \hbar}{2 m \omega}\left[\left(\tilde{X}_{x_{1}}^{L}\right)^{2}+\left(\tilde{X}_{x_{2}}^{L}\right)^{2}\right]\right\} \Psi=0$, for the attractive harmonic oscillator, and $\left\{\tilde{X}_{B}^{L}+\tilde{X}_{C}^{L}+\frac{i \hbar}{2 m \omega}\left[\left(\tilde{X}_{x_{1}}^{L}\right)^{2}-\left(\tilde{X}_{x_{2}}^{L}\right)^{2}\right]\right\} \Psi=0$ for the repulsive harmonic oscillator.

We shall not solve these polarization equations, see, for instance, [41] and [42], for the description of the harmonic oscillator in configuration space.

Finally, we would like to make few comments on the geometric interpretation of the anomaly of the Schrödinger group. As commented at the beginning of this section, the classical description of this group leads to a system with one degree of freedom, associated essentially to the coadjoint orbits (for each value of the parameter $\frac{m \omega}{\hbar}$ ) of the Heiserberg-Weyl subgroup. That is, the $S L(2, R)$ subgroup, lying in the kernel of the symplectic form, is represented trivially; we are in the trivial (the identity of $\left.s l(2, R)^{*}\right)$ coadjoint orbit of $S L(2, R)$. Therefore, the classical description has the same features as for any other semidirect product, like for instance, the action of spatial rotations on the Galilei or the Poincaré groups (without spin).

However, at the quantum level, meanwhile for the Galilei or Poincaré groups first-order (full and symplectic) polarizations can be found (see [26]), that is, they are not anomalous groups, for the Schrödinger group we have to resort to a higher-order polarization to obtain the representation associated with only one degree of freedom. Computing the Casimir operator for the $S L(2, R)$ subgroup,

$$
\hat{C} \equiv \frac{1}{4}\left(\tilde{X}_{A}^{R}-\tilde{X}_{D}^{R}\right)^{2}+\frac{1}{2}\left(\tilde{X}_{B}^{R} \tilde{X}_{C}^{R}+\tilde{X}_{C}^{R} \tilde{X}_{B}^{R}\right),
$$

it is easy to check that the representation obtained contains two irreducible representations of $S L(2, R)$, corresponding to Bargmann's indices $k=\frac{1}{4}$ and $\frac{3}{4}$, and the Casimir operator turns out to be $\hat{C} \Psi=-\frac{3}{16} \Psi$ (the relation between the value $c$ of the Casimir and the Bargmann's index $k$ is given by $c=k(k-1)$. This means that to construct the quantum representation associated with the trivial coadjoint orbit of $S L(2, R)$ one must use two different coadjoint orbits, none of them being the trivial one. Thus, the Schrödinger group is the simplest example for which the corresponce between coadjoint orbits and quantum Hilbert spaces is broken. Another example is that of the Virasoro group, which will be discussed in next section. 
An interesting physical application of this construction can be found in Quantum Optics -in two-photon systems 43]- where the action of the generators of the $S L(2, R)$ subgroup represent the emission or absorbtion of two photons simultaneously. The two irreducible representations of $S L(2, R)$ with $k=\frac{1}{4}$ and $\frac{3}{4}$ correspond to the set of states with even and odd number of photons, respectively, and both sets are needed to construct an irreducible representation of the whole Schrödinger group.

\subsection{The Virasoro group and String Theory}

Let us comment briefly on the relevant, although less intuitive, example of the infinite-dimensional Virasoro group. Its Lie algebra can be written as

$$
\left[\tilde{X}_{l_{n}}^{L}, \tilde{X}_{l_{m}}^{L}\right]=-i(n-m) \tilde{X}_{l_{n+m}}^{L}-\frac{i}{12}\left(c n^{3}-c^{\prime} n\right) \Xi,
$$

where $c$ parametrizes the central extensions and $c^{\prime}$ the pseudo-extensions. For the particular case in which $\frac{c^{\prime}}{c}=r^{2}, r \in N, r>1$, the co-adjoint orbits admit no invariant Kählerian structure, as was firstly stated by Witten pointing out a breakdown of Geometric Quantization. In the present approach, this case shows up as an algebraic anomaly. In fact, the characteristic subalgebra is given by

$$
\mathcal{G}_{C}=<\tilde{X}_{l_{0}}^{L}, \tilde{X}_{l_{-r}}^{L}, \tilde{X}_{l_{+r}}^{L}>,
$$

which is not fully contained in the non-full (but symplectic) polarization

$$
\mathcal{P}^{(r)}=<\tilde{X}_{l_{n \leq 0}}^{L}>\text {. }
$$

There also exists a full polarization $\mathcal{P}_{C}=<\tilde{X}_{l_{k r}}^{L}>, r>1, k=-1,0,1,2,3, \ldots$ which is not symplectic since none of the symplectic generators with labels $l_{ \pm r^{\prime}}, r^{\prime} \neq k r$ are included in the polarization. A detailed description of the representations of the Virasoro group can be found in [24] and references therein.

The situation is formally similar to that found in the Schrödinger group. Now, for particular values of the parameters $c, c^{\prime}$ or, equivalently, $c, h \equiv \frac{c-c^{\prime}}{24}$ given by the Kac formula [44],

$$
\begin{aligned}
h= & \frac{1}{48}(13-c)\left(k^{2}+s^{2}\right) \pm\left(c^{2}-26 c+25\right)\left(k^{2}-s^{2}\right) \\
& -24 k s-2+2 c \\
& k, s \text { positive integers, } k s \leq r,
\end{aligned}
$$


the "quantum values" of the anomaly, the representations given by the first order non-full (symplectic) polarizations (120) are reducible since there exist invariant subspaces characterized by certain higher-order polarization equations [24]. On these subspaces the operators $<\tilde{X}_{l_{0}}^{R}, \tilde{X}_{l_{-r}}^{R}, \tilde{X}_{l_{+r}}^{R}>$, the right version of the characteristic subalgebra (119), can be rewritten in terms of the basic operators $\tilde{X}_{l_{k}}^{R}, k \neq \pm r, 0$. There is a (standard) anomaly in the sense that the classical symplectic manifold is reduced (the Noether invariants associated with $<\tilde{X}_{l_{0}}^{R}, \tilde{X}_{l_{-r}}^{R}, \tilde{X}_{l_{+r}}^{R}>$ are written in terms of the basic ones) for the value $c^{\prime}=c r^{2}$ or, equivalently, $h=-c\left(r^{2}-1\right) / 24$, instead of the values given by (121). Note that there is no one-to-one correspondence between the values of $c^{\prime} / c$ characterizing the coadjoint orbits of the Virasoro group (the classical values of the anomaly) and the values allowed by the Kac formula (the quantum values of the anomaly), a fact which must be interpreted as a breakdown of the notion of classical limit.

String theory provides another example of anomalous system with characteristics maybe more similar to the finite-dimensional case. The symmetry of the bosonic string consists of an infinite set of harmonic oscillators, the normal modes, on which the Virasoro group acts by semi-direct product. That is,

$$
\begin{aligned}
{\left[\tilde{X}_{l_{n}}^{L}, \tilde{X}_{l_{m}}^{L}\right] } & =-i(n-m) \tilde{X}_{l_{n+m}}^{L}-i \frac{1}{12}\left(c n^{3}+c^{\prime} n\right) \delta_{n+m, 0} \tilde{X}_{0}^{L} \\
{\left[\tilde{X}_{l_{n}}^{L}, \tilde{X}_{\alpha_{m}^{\mu}}^{L}\right] } & =-i m \tilde{X}_{\alpha_{n+m}^{\mu}}^{L} \\
{\left[\tilde{X}_{\alpha_{n}^{\mu}}^{L}, \tilde{X}_{\alpha_{m}^{\nu}}^{L}\right] } & =-i a m \delta_{n+m} \delta^{\mu \nu} \tilde{X}_{0}^{L}
\end{aligned}
$$

The anomaly is clearly discovered when considering the specific values $c=$ $0=c^{\prime}$ of the extension parameters, corresponding to the classical symmetry. For these values the characteristic subalgebra is

$$
<\tilde{X}_{\alpha_{0}^{\mu}}^{L}, \tilde{X}_{l_{n}}^{L}>, n \in Z
$$

and the only allowed polarization,

$$
\mathcal{P}=<\tilde{X}_{\alpha_{n \leq 0}^{\mu}}^{L}, \tilde{X}_{l_{n \leq 0}}^{L}>
$$

is non-full, excluding the Virasoro generators with positive index, which also are in the characteristic subalgebra. Quantizing with this polarization leads to a Hilbert space containing states obtained from the vacuum by the action 
of all the creation operators, i.e. $<\tilde{X}_{\alpha_{n>0}^{\mu}}^{L}, \tilde{X}_{l_{n>0}}^{L}>$. However, for the "quantum" values of the anomaly, $c=d=c^{\prime}$, where $d$ is the dimension of the Minkowski space, the Hilbert space is generated only by states of the form

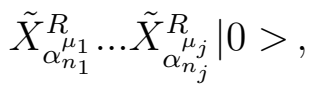

and the Virasoro operators are all of them written in terms of the true basic operators, according to the Sugawara's construction [45]:

$$
\tilde{X}_{l_{k}}^{R}=\frac{1}{2} g_{\mu \nu}: \sum_{n} \tilde{X}_{\alpha_{k-n}^{\mu}}^{R} \tilde{X}_{\alpha_{n}^{\nu}}^{R}:
$$

This process is essentially equivalent to the anomalous reduction which allows the $\operatorname{sl}(2, R)$ operators to be written in terms of $\tilde{X}_{x_{1}}^{R}, \tilde{X}_{x_{2}}^{R}$ in the case of the Schrödinger group.

\section{References}

[1] van Hove, J.: Acsd. R. Belg., Cl. Sci., Mem., Collect., 6, t. XXVI (1951)

[2] Aldaya, V. and de Azcárraga, J.: J. Math. Phys. 23, 1297 (1982)

[3] A.A. Kirillov, Elements of the Theory of Representations, SpringerVerlag, Berlin-Heidelberg-New York (1976)

[4] B. Kostant, Quantization and Unitary Representations, in Lecture Notes in Mathematics 170, Springer-Verlag, Berlin (1976)

[5] J.M. Souriau, Structures des systemes dynamiques, Dunod, Paris (1970)

[6] Pressley A., and Segal, G.: Loop Groups, Clarendon Press, Oxford (1986)

[7] P. Malliavin, Geometrie differentielle intrinseque, Hermann, Paris (1969)

[8] C. Godbillon, Geometrie differentielle et mechanique analytique, Hermann, Paris (1969) 
[9] R. Abraham and J.E. Marsden, Foundations of Mechanics, BenjaminCummins, New York (1978); G. Marmo, E. J. Saletan, A. Simoni, B. Vitale, Dynamical Systems, a Differential Geometric Approach to Symmetry and Reduction, J. Wiley, New York (1985)

[10] S. Kobayashi and K. Nomizu, Foundations of Differential Geometry, Interscience, New York, Vol. I (1963)

[11] Woodhouse, N., Geometric Quantization, Clarendon, Oxford (1980)

[12] J.M. Lévy-Leblond, in Group Theory and its Applications, Vol. 2, E.M. Loebl, Academic Press, New York (1971)

[13] E.C.G. Sudarshan and N. Mukunda, Classical Dynamics: A Modern Perspective, J. Wiley, New York (1974); G. Marmo, G. Morandi, A. Simoni and E.C.G. Sudarshan, Phys. Rev. D37, 2196 (1988); G. Marmo, E.J. Saletan and A. Simoni, Il Nuovo Cimento 96B, 159 (1988); G. Marmo, G. Morandi and C. Rubano, Symmetries in the Lagrangian and Hamiltonian Formalism: The Equivariant Inverse Problem, in Symmetries in Science III (B. Gruber, F. Iachello Eds.), Plenum Press, New York (1988)

[14] V. Aldaya and J.A. de Azcárraga, Riv. Nuov. Cim. 3 (10), 1 (1980)

[15] V. Bargmann, Ann. Math. 59, 1 (1954)

[16] A.G. Kurosh, The Theory of Groups, Vol. II, Chelsea, New York (1960)

[17] L. Michel, in Group Theoretical Methods in Elementary Particles, F. Gursey ed., Gordon and Breach, New York (1964)

[18] Saletan, E.J.: J. Math. Phys. 2, 1 (1961)

[19] Aldaya, V. and de Azcárraga, J.A.: Int. J. Theo. Phys. 24, 141 (1985)

[20] E. Inönü and E.P. Wigner, Proc. Natl. Acad. Sci. U.S.A., 39, 518 (1953)

[21] E. Inönü, in Istambul Summer School of Theoretical Physics, F. Gürsey, ed., Gordon and Breach, New York (1962)

[22] N. Jacobson, Lie Algebras, Dover Publications, Inc, New York (1979) 
[23] Aldaya, V. and Navarro-Salas, J.: Commun. Math. Phys. 113, 375 (1987)

[24] Aldaya, V. and Navarro-Salas, J.: Commun. Math. Phys. 139, 433 (1991)

[25] Aldaya, V., Navarro-Salas, J., Bisquert, J. and Loll, R.: J. Math. Phys. 33, 3087 (1992)

[26] Aldaya, V., Bisquert, J., Guerrero, J. and Navarro-Salas, J. : J. Phys. A26, 5375 (1993)

[27] Aldaya, V., Navarro-Salas, J. and Ramírez, A.: Commun. Math. Phys. 121, 541 (1989)

[28] Aldaya, V., Calixto, M. and Guerrero, J.: Commun. Math. Phys. 178, 399 (1996)

[29] Balachandran, A.P., Marmo, G.,Sakgerstam, B.S. and Stern, A., Classical Topology and Quantum States, World Scientific, Singapore, (1991)

[30] V. Aldaya, J. Guerrero and G. Marmo, Int. J. Mod. Phys. A12, 3 (1997)

[31] Aldaya, V., de Azcarraga, J.A. and Wolf, K.B., J. Math. Phys. 25, 506 (1984)

[32] M. Navarro, V. Aldaya and M. Calixto, J. Math. Phys. 28, 1454 (1997)

[33] Fernández, M., Gotay, M.J. and Gray, A.: Proc. Am. Math. Soc. 103, 1209 (1988)

[34] Jackiw, R. In: Current Algebra and Anomalies, Treiman, S.B., Jackiw, R., Zumino, B. and Witten, E. (eds.) World Scientific, Singapore (1985)

[35] Serre, J.P.: Lie Algebras and Lie Groups, W.A. Benjamin, INC, New York (1965)

[36] Wigner, E.P.: Group Theory, Academic Press (1959)

[37] Niederer, U.: Helv. Phys. Acta 45, 802 (1972); 46, 191 (1973); 47, 167 (1974) 
[38] M. Perroud, Helv. Phys. Acta 50, 233 (1977)

[39] M. Calixto, V. Aldaya and J. Guerrero, Generalized Conformal Symmetry and Extended Objects from Elementary Systems, in preparation.

[40] G. B. Folland, Harmonic Analisys in Phase Space, Ann. Math. Studies 122. Princenton University Press, Princenton (1989)

[41] V. Aldaya, J. Bisquert, J. Guerrero and J. Navarro-Salas, Rep. Math. Phys. 37, 387 (1996)

[42] V. Aldaya, J. Guerrero and G. Marmo, Higher-order Quantization on a Lie Group, hep-th/9512020, (submitted to J. Math. Phys.) (1995)

[43] Yuen, H.P.: Phys. Rev. A 13, 2226 (1976)

[44] Kac, V.G.: Lecture Notes in Physics 94, 441. Berlin, Heidelberg, New York: Springer (1982)

[45] H. Sugawara, Phys. Rev. 170, 1659 (1968) 